\title{
STOCHASTIC DYNAMICS OF MICROCAVITY POLARITONS
}

Thesis for:

Master Degree in Physics

\author{
Author: \\ Alvaro Enrique Pedraza \\ Physics Department \\ Universidad de los Andes \\ Thesis Advisor: \\ Luis Quiroga \\ Physics Department \\ Universidad de los Andes
}

Universidad de los Andes

Physics Department

BOGOTA, COLOMBIA

August, 2006 


\begin{abstract}
We study the time dependent polariton condensation as well as the parametric scattering process of polaritons in a semiconductor microcavity. Based upon a new stochastic scheme the dynamics for both cases is fully analyzed. We show how the evolution of the system is described by a set of stochastic differential Schrodinger equations which in average reproduces the exact dynamics. Furthermore, we underline the role that Coulomb correlations plays in the polariton dynamics and show a spontaneous coherence builup driven by polariton-polariton interactions. Threshold behaviors are well captured by the present approach and the results are in complete agreement with recent experimental observations.
\end{abstract}




\section{Contents}

1 Introduction 3

2 Theory of Microcavity Polaritons 5

2.1 The basic Hamiltonian for excitons and photons in the strong coupling regime . . . . 6

2.2 Non linear effects and inter-particle interactions . . . . . . . . . . . . 8

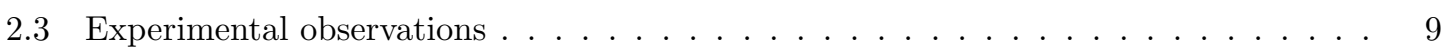

2.4 Coupling with the semiconductor environment $\ldots \ldots \ldots \ldots \ldots$

3 Exact dynamics of interacting systems: Stochastic approach $\quad 15$

3.1 N-Boson system with two-body interaction. Closed system . . . . . . . . . . . . 16

3.1.1 Stochastic formulation of a N-Boson closed system . . . . . . . . . . . . 16

3.1.2 Evolution of the single particle state . . . . . . . . . . . . . . . . 19

3.1.3 Real time evolution . . . . . . . . . . . . . . . . . . . . . 21

3.1.4 Imaginary time evolution: Thermal equilibrium . . . . . . . . . . . . . . . . 24

3.2 Exact formulation for open quantum systems $\ldots \ldots \ldots \ldots \ldots \ldots$

3.2.1 Diffusive stochastic wave function approach $\ldots \ldots \ldots \ldots \ldots \ldots$

3.2 .2 Exact and stochastic dynamics $\ldots \ldots \ldots \ldots \ldots \ldots$

3.2 .3 Determination of the observables . . . . . . . . . . . . . . . . 30

3.2 .4 Important features of the method . . . . . . . . . . . . . . . . . . 30 
3.2 .5 The Jaynes-Cummings model . . . . . . . . . . . . . . . . . . . . . . . . 31

3.2.6 Two level system (qubit) coupled with a thermal bath . . . . . . . . . . 36

3.3 Exact reformulation of interacting bosons in the presence of an environment . . . . . 37

3.3 .1 Stochastic solution . . . . . . . . . . . . . . . . . . . . . . . 39

3.3.2 N-bosons in a two level system coupled with an external mode . . . . . . . . 41

4 Spontaneous coherence build-up and dynamics of Bose-Einstein phase transition in microcavity polaritons

4.1 Dynamical condensation of microcavity polaritons . . . . . . . . . . . . 45

4.2 Parametric down convertion . . . . . . . . . . . . . . . . . . . . 51

5 Conclusions $\quad 56$ 


\section{Chapter 1}

\section{Introduction}

Microcavity polaritons are quasiparticles created in the strong coupling regime between photons in a microcavity and the excitonic resonance of a semiconductor quantum well [1]. Polaritons satisfy Bose statistics (at least in the low density regime) and are excellent candidates to study quantum effects at the macroscopic level, e.g. Bose-Einstein Condensation (BEC) and superfluidity. As a practical advantage that polariton systems exhibit over other quasi-bosonic particles in condensed matter systems is their easy experimental optical control $[2,3]$. Particulary, polaritons can be optically injected in the microcavity by external light impinging on the semiconductor nanostructure, and their properties can be inferred from the observation of the emitted light. The possibility of obtaining a quantum fluid in a solid state system, with easy control and integration potentialities, could also open a new promising way to the implementation of quantum information technologies.

The polariton condensation has being actively pursued, since no BEC has being observed for a solid state system. Recently, a possible quantum phase transition of polaritons was suggested [4], and the spontaneous formation of a coherent polariton state was reported in [5]. This last result shows a remarkable manifestation of the bosonic behavior of this short living particles, but it has been interpreted as a polariton laser phase transition. From the theoretical side, most existing works [4] pursue a strict analogy with the laser theory, where the quantum fluctuations responsible for the phase transition appear directly from the particle number conservation.

On the other hand, it is well known that due to their excitonic component, polaritons are subjected to Coulomb interactions. This nonlinearity is expected to trigger the quantum phase transition. Moreover, the unexpected observation of a thermal-like intensity far above the threshold [5] does not match with the laser picture, leaving the unanswered question of the role of interactions, usually overlooked in the theory. A more recent attempt to address this question has been made in [6], where the laser picture was left behind and polariton-polariton interactions were taken into account. 
Nonetheless, this last study is within the scope of a mean-field approximation (MFA), and thus fails to give a proper description of the polariton dynamics above the parametric threshold where high order Coulomb correlations are more important than in the low density regime.

The present document is devoted to gain more physical insight into the polariton dynamics by analyzing two different dynamical processes: (i) the time-dependent condensation and coherence build-up of polaritons relaxing into the lowest branch states and (ii) the time-dependent parametric scattering. Both processes will be considered within the same theoretical approach based on the stochastic unravelling of the dynamics of an interacting many-body system embedded in a noisy environment. Partial realizations of such a computational scheme have been previously considered. On the one hand, closed quantum interacting systems have been studied by stochastic unravellings in Ref.[7] for bosons and in Ref.[8] for fermions. On the other hand, simple open quantum systems have been studied by following the stochastic density matrix for the whole system plus environment in Refs.[10,11]. It is one of the main aims of the present work to extend those partial developments to a complex many-body open quantum system such as the one offered by microcavity polaritons. In order to provide quantitative predictions, previous stochastic schemes are extended to follow the evolution of a polariton system coupled to a noisy environment where interparticle interactions and environment effects can be treated on the same footings. This will allow us to transform the evolution of an open interacting polariton system, into the stochastic evolution of a single polariton [7] separately from the stochastic evolution of the environment [10, 11]. As compared with similar approaches the advantages of this stochastic scheme are manifold: first of all, the complete dynamics, both transient and steady-state regimes, can be obtained with full fluctuations due to interactions properly taken into account. Second, a many-polariton problem can be mapped onto a single polariton system driven by stochastic terms.

The document is organized as follows: The basic theory of microcavity polaritons is presented in Chapter 2. In Chapter 3 a detailed description of the stochastic scheme is presented. In Chapter 4.1 the polariton condensation dynamics with both Coulomb interactions and phonon scattering is considered. We pay particular attention to the quantum properties of the condensate fraction and the second-order degree of coherence. The parametric scattering of pump polaritons in the lower polariton branch and the dynamics of formation of the eight-shaped final allowed states is studied in Chapter 4.2, where comparison to experimental results [2] reveals the importance of including the nonlinearities associated to polariton interactions, specially above the threshold regime. Finally, in Chapter 5 we sketch our concluding remarks. 


\section{Chapter 2}

\section{Theory of Microcavity Polaritons}

In recent years the nonlinear optical properties of low-dimensional semiconductor structures have been the subject of a large number of investigations [12, 13, 14], specially in the strong coupling regime. As mentioned before, microcavity polaritons are two-dimensional particles with exotic properties and ease of optical control. They differ from the bulk polaritons which cannot be directly excited by optical means [13]. Due to their cavity photon component, they have a very sharp energy dispersion and its effective mass is four orders of magnitude lighter than those of the quantum well excitons. Their nonlinear behavior is produced by the exciton-exciton Coulomb interaction and their bosonic behavior survives as long as the excited density is much smaller than the typical excitonic density. Thus, high temperature two dimensional BEC is expected [15]. Other quantun effects such as squeezing and quantum correlations should rise from the coherent nature of microcavity polaritons.

Given this general properties, most autors have considered microcavity polaritons as ideal for the experimental study of nonlinear dynamics of interacting particles. One of the pioneer works on this subject was [16], where a giant amplification was shown above some threshold on the driving field. In this type of experiment, polaritons are optically excited at the desired energy and momentum allowing direct control on their dynamics. More recently, evidence on the existence of polariton pair correlations were shown through the interference of the emission from different polariton states [3]. This result has open the possibility of producing many-particle entangled states of light-matter waves.

From a theoretical point of view we want to focus on the dynamical behavior of microcavity polaritons. In this Chapter we will guideline the general theory of microcavity polaritons. Starting from the effective Hamiltonian for the coupled exciton-photon system, we will construct the microcavity polariton states and descrice the polariton-polaritons interactions as well as the coupling with 
the semiconductor environment. The baseline model and the relevant parameteres will be introduced. Eventhough this are straightforward results discussed extensively in the literature, they are the divergence point between previous studies on the dynamical behavior of this systems and our stochastic approach. While some autors favor semiclassical Boltzmann rate equations $[15,17,18]$ and others MF approximations [6, 12, 13], we will develop a stochastic scheme (Chapter 3) that recovers the complete dynamics of many-body systems such as microcavity polaritons

\subsection{The basic Hamiltonian for excitons and photons in the strong coupling regime}

We consider a microcavity containing a semiconductor quantum well embedded between two highly reflecting planar Bragg mirros separated by a distance of the order of the wave-length [14]. The discussion is limited to a two-band semiconductor. The electromagnetic field can excite an electron from the filled valence band to the conduction band, thereby creating a hole in the valence band. The electron-hole system possesses bound states, the excitonic states. The excitons are confined along the growth direction ( $z$ direction) and free in the plane of the layers ( $x y$ plane). The excitons couple with the light field in the cavity.

Neglecting the spin degrees of freedom, we can write an effective interaction Hamiltonian for the coupled exciton-photon system as

$$
H=\sum_{k} E_{e x}(k) b_{k}^{\dagger} b_{k} \sum_{k} E_{c a v}(k) c_{k}^{\dagger} c_{k}+\sum_{k} \frac{\hbar \Omega_{R}}{2}\left(b_{k}^{\dagger} c_{k}+b_{k} c_{k}^{\dagger}\right)
$$

with the sum taken over the set of plane wave solutions in a two-dimensional box. The first two terms correspond to the free energies of excitons and photons, and $b_{k}$ and $c_{k}$ are the annihilation operators of excitons and photons respectively. In Hamiltonian $(2.1) E_{c a v}(k)$ is the dispersion relation of the confined photon,

$$
E_{c a v}(k)=\sqrt{E_{c a v}(0)+\frac{\hbar^{2} c^{2} k^{2}}{n^{2}}}
$$

where $c$ is the speed of light and $n$ the refractive index of the cavity. $E_{e x}$ is the dispersion of the exciton

$$
E_{e x}(k)=E_{e x}(0)+\frac{\hbar^{2} k^{2}}{2 m_{e x}}
$$


with $E_{e x}(0)$ as the rest energy of the bound state hole-electron, and $m_{e x}$ its effective mass. Here $m_{e x}$ is so large that the dispersion is almost flat over the range of wavevectors of interest. Following [17] one can treat excitons as bosons in the low density regime $n a_{0}<<1$. In such an approximation, the linear Hamiltonian can be easily diagonalized in terms of another bosonic operator which can be written as

$$
\begin{gathered}
p_{k}^{-}=X_{k} b_{k}-C_{k} c_{k} \\
p_{k}^{+}=C_{k} b_{k}+X_{k} c_{k} .
\end{gathered}
$$

Hence Hamiltonian (2.1) reduces to free propagation terms only

$$
H=\sum_{k} E_{+}(k) p_{k}^{+\dagger} p_{k}^{+}+\sum_{k} E_{-}(k) p_{k}^{-\dagger} p_{k}^{-}
$$

for upper $(+)$ and lower $(-)$ branches energies

$$
E_{k}^{ \pm}=\frac{1}{2}\left(E_{e x}(k)+E_{c a v}(k) \pm \hbar \sqrt{\delta_{k}^{2}+\Omega_{R}^{2}}\right),
$$

where $\delta_{k}$ is the detunning between the cavity and exciton modes, $\delta_{k} \equiv E_{c a v}(k)-E_{e x}(k)$. Within this simple treatment, polaritions are the resulting two-dimensional eigenstates from the mixing of photon-exciton states, while the exciton and photon modes can be interpreted as transient states. This theoretical description is sketched in Figure 2.1, where the total enenergy dispersion of the polariton is plotted and compare with the individual energy dispersion of the cavity photon and exciton. Finally, the coefficients used to diagonilize (2.1) are

$$
C_{k}^{2}=\frac{\Omega_{R}^{2}}{2 \sqrt{\delta_{k}^{2}+\Omega_{R}^{2}}\left(\delta_{k}^{2}+\sqrt{\delta_{k}^{2}+\Omega_{R}^{2}}\right)}
$$

and

$$
X_{k}^{2}=\frac{\delta_{k}^{2}+\sqrt{\delta_{k}^{2}+\Omega_{R}^{2}}}{2 \sqrt{\delta_{k}^{2}+\Omega_{R}^{2}}} .
$$

These are the so called Hopfield coefficients $[13,14,17]$. They represent the exciton $\left(X_{k}\right)$ and photon fraction $\left(C_{k}\right)$ of the polariton. Experimental measurement of Hopfield coefficients and 


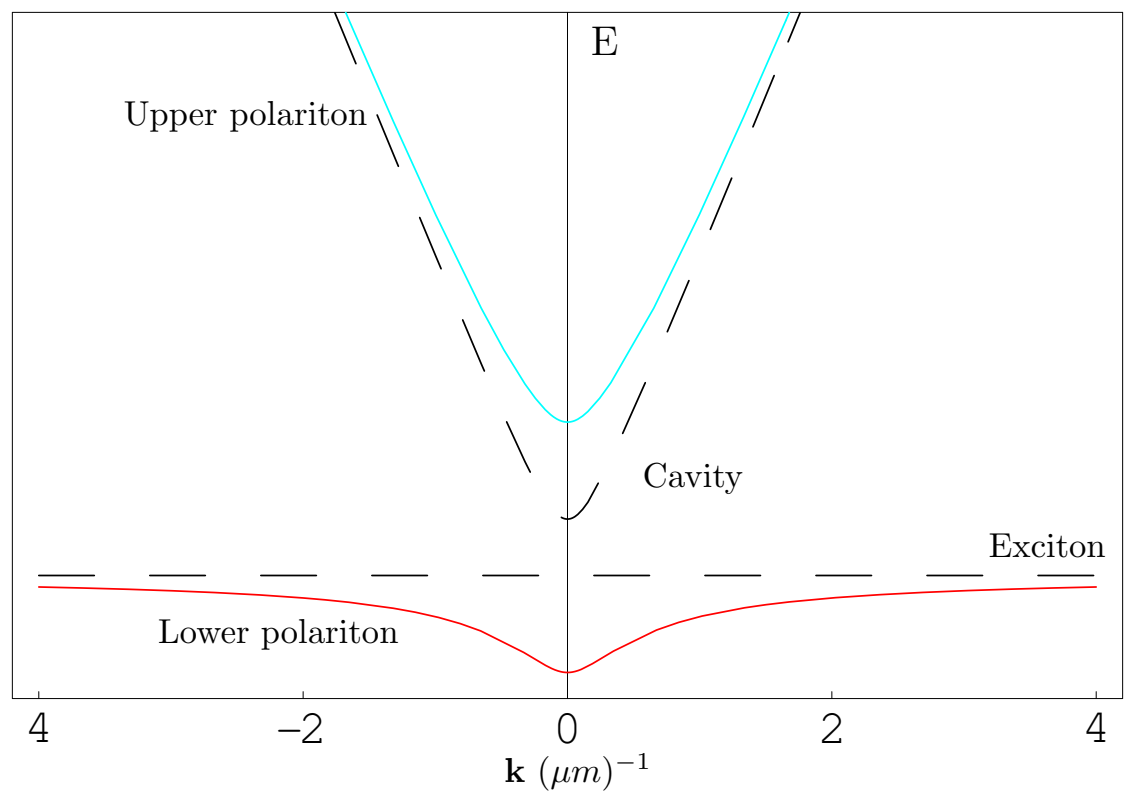

Figure 2.1: The solid lines depict the energy dispersion of the lower polariton (red) and upper polaritons (blue) branches. The dashed lines describe the bare quantum well exciton and cavity photon dispersions.

Hopfield theory was done in detail in [19]. In the following we will limit ourselves to the polariton basis 2.4, and more specifically to the lower polariton branch, were most of the interesting behavior takes place. In particular, in the experiments we are interested in modeling the pump is spectrally narrow and resonantly excites the lower polariton branch. Thus, we dropped the $(-)$ and $(+)$ upper indexes and the free Hamiltonian of polaritons in the lower branch is written as $H=\sum_{k} E(k) p_{k}^{\dagger} p_{k}$.

\subsection{Non linear effects and inter-particle interactions}

Polaritons can scatter with each other thanks to their underlying excitonic fraction, of the dipoledipole interaction type. This effect becomes noticeable when the number of polaritons is increased. In more detail, the effect of the collision between two excitons gives rise to two other excitons. The effective Hamiltonian for this exciton-exciton interaction reads

$$
H_{X X}=\frac{1}{2} \sum_{k, k^{\prime}, q} V_{q}^{X X} b_{k+q}^{\dagger} b_{k^{\prime}-q}^{\dagger} b_{k} b_{k^{\prime}}
$$

where $V_{q}^{X X}$ is the effective interaction potential which is determined from the Coulomb electron-hole Hamiltonian [13]. For small wave vector, $V_{q}^{X X}$ has a weak dependence on $q$, and the interaction 
potencial can be written as $V_{q}^{X X} \simeq V_{0}^{X X}$, which is a contact potential. This approximation is widely used $[14,16]$ because resonantly excited excitons have very small wave vectors. The zero wave vector value of the effective potential is $V_{0}^{X X}=\frac{6 e^{2} \lambda_{x}}{A \epsilon}$, where $\lambda_{x}$ is the exciton radius, $\epsilon$ the dielectric constant of the quantum well and $A$ the macroscopic quantization area.

Also, since the exciton quasi-particle is a composite boson formed by a par of fermions, due to this fermionic components, the exciton-photon coupling is saturable [13]. This gives rise to an anharmonic interaction term whose Hamiltonian form is

$$
H_{X C}^{s a t}=-\sum_{k, k^{\prime}, q} \frac{\hbar \Omega_{R}}{n_{s a t} A} a_{k+q}^{\dagger} b_{k^{\prime}-q}^{\dagger} b_{k} b_{k^{\prime}}+\text { h.c. }
$$

where $n_{\text {sat }}=7 /\left(16 \pi \lambda_{x}^{2}\right)$ is the exciton saturation density.

In order to rewrite the exciton-exciton interaction Hamiltonian (2.10) and the anharmonic photonexciton coupling (2.11) in terms of polariton operators, we need to recall (2.4). It is straightforward to note that the cavity photon and the exciton operator can be written as $a_{k}=C_{k} p_{k}^{-}+X_{k} p^{+}$and $b_{k}=X_{k} p_{k}^{-}-C_{k} p^{+}$respectively. Replacing this expressions in (2.10) and (2.11) and dropping the terms containing operators from the upper polariton branch from the reasons mention before, the Hamiltoninan containing the non-linear terms in the lower polation branch is then

$$
H_{P P}=\frac{1}{2} \sum_{k, k^{\prime}, q} \frac{\lambda_{X}^{2}}{A} V_{k, k^{\prime}, q}^{P P} p_{k+q}^{\dagger} p_{k^{\prime}-q}^{\dagger} p_{k} p_{k^{\prime}}
$$

This is the effective polariton-polariton interaction. Where the interaction potential is $[12,13]$

$$
V_{k, k^{\prime}, q}^{P P}=\frac{6 e^{2}}{\epsilon \lambda_{X}} X_{k+q} X_{k^{\prime}} X_{k^{\prime}-q} X_{k}+2 \frac{\hbar \Omega_{R}}{n_{s a t} \lambda_{X}^{2}}\left(\left|C_{k+q}\right| X_{k^{\prime}}+\left|C_{k^{\prime}}\right| X_{k+q}\right) X_{k^{\prime}-q} X_{k}
$$

Finally, one should read Hamiltonian (2.12) as the resulting scattering process from a pair of polaritons in the momentum space. Evidence of this nonlinear process has being recently reported and will be the topic of the next section.

\subsection{Experimental observations}

For resonant polariton excitation, polaritons are created in a well defined $\mathbf{k}[2,3]$ in the momentum space. The parametric scattering which arises from polariton-polariton interactions results in a 


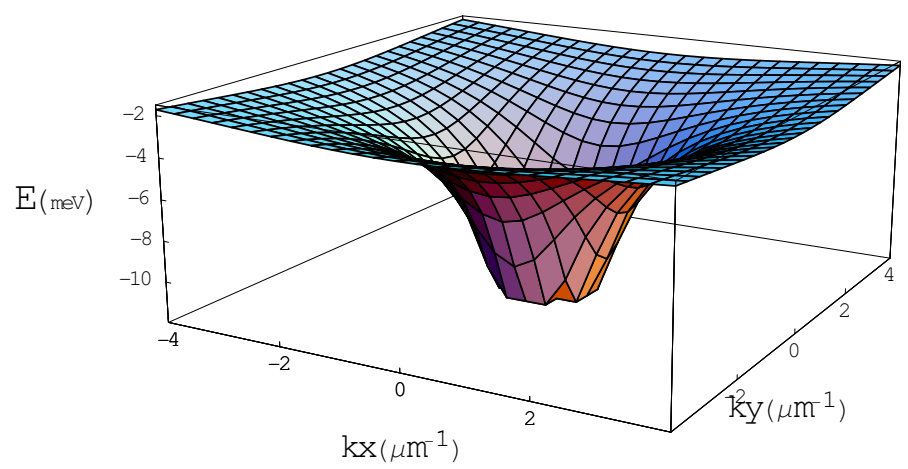

Figure 2.2: Lower polariton energy dispersion versus $k_{x}$ and $k_{y}\left(\mu m^{-1}\right)$.

spontaneous emission of polariton pairs, signal and idler. This scattering is restricted to energy and momentum conservation rules. The peculiar inverse "Mexican-hat" structure from the energy dispersion in the lower polariton branch (see Figure 2.2) allows the energy and momentum conserving pair of polaritons to scatter on an 8-shaped line in the $\mathbf{k}$ space. The first theoretical description of this process was done by Ciuti et al in [12].

This scattering mechanism can be modeled as follows. Let us consider a pair of polariton pumped directly at the same wave vector $k_{p}$. The resulting pair, which are called individually signal $\left(k_{s}\right)$ and $\left(k_{i}\right)$ idler polaritons with $\left|k_{s}\right|<\left|k_{i}\right|$, have to fulfill the energy conservation condition $2 E\left(k_{p}\right)=$ $E\left(k_{s}\right)+E\left(k_{i}\right)$. The posible final states resulting from the parametric scattering from a given pump are ploted in figure (2.3). This is a symetric 8-shape in the signal and idler states. The relevant material parameters [2] are typical for a GaAs-based microcavity: heavy-hole exciton Rabi splitting $\hbar \Omega_{R}=1.82 \mathrm{meV}$, the detunning with the confined photon mode $-2.4 \mathrm{meV}$ and the effective index of the cavity mode 3.5. This parameters will be used troughout this document.

Although this simple description is very useful, and is usually the starting point for parametric scattering studies [2,3]; it is only a first order approximation on the dynamics due to the action of Hamiltonian (2.12). More precisely, some extra sattering processes specially from the idler sates are also present. This is a consecuence from the well know fact that high momentum polaritons, have a larger fraction of excitonic component. Thus higher Coulomb interaction. Previous works have model this correlations through Langevin equations [12]. The first experimental observation on the predicted figure- 8 shaped (in the momentum space) distribution of the final states from the parametric scattering was reported by [2]. As discussed, the influence of higher-order scattering is crucial on the dynamics. In particular, the emission from the idler states is asymetric from the signal one. While signal states show a closer resemblance to the predicted in the first order scattering process, the idler states are clearly different and asyncronic. In figure 2.4 experimental observations from this processes are depicted. A clear result from this work, Langbein [2] showed 

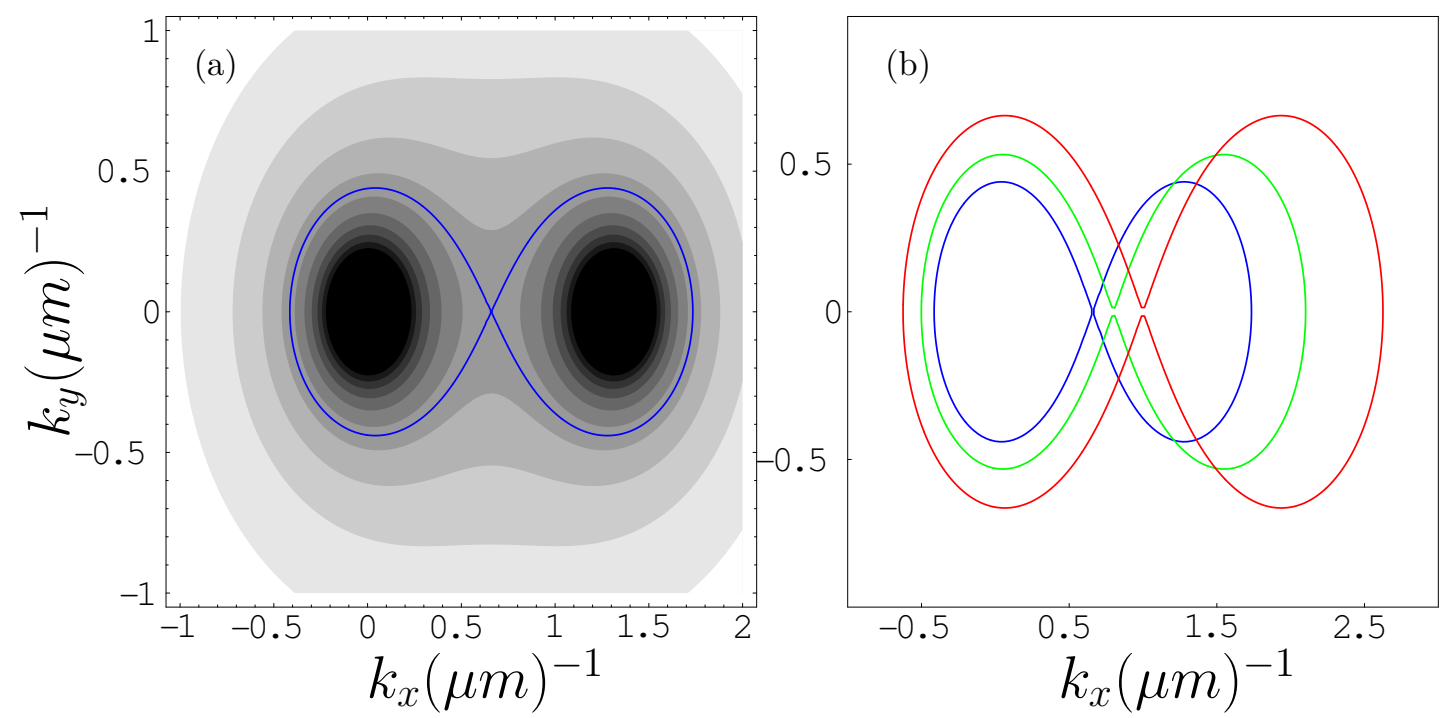

Figure 2.3: (a) Contour plot of the energy balance equation $\left|E_{k_{s}}+E_{k_{i}}-2 E_{k_{p}}\right|$. (b) Final states for two pump polaritons at $\mathbf{k}_{\mathbf{p}}=\{0.66,0\} \mu m^{-1}$ (blue), $\mathbf{k}_{\mathbf{p}}=\{0.8,0\} \mu m^{-1}$ (green) and $\mathbf{k}_{\mathbf{p}}=$ $\{0.1,0\} \mu m^{-1}$ (red) spontaneously scattered conserving both energy and momentum

that multiple scattering are of significant importance even for small excitation intensities.

A previous experimental achivement from polariton parametric scattering was the so called polariton parametric amplifier. This is obtained by a two-beam pump-probe scheme [16]. While a weak probe stimulates the lower branch at $k=0$, a pump beam resonantly excites the lower polariton branch at $k_{p}$. When an intense enough pump beam excites the sample at the wave vector $k_{p}$ which exactly fulfills the condition $2 E\left(k_{p}\right)=E(0)+E\left(2 k_{p}\right)$, a large amplification of the probe beam is obtained. Similar to the previous discussion, a pair of polaritons is scatter by means of Coulomb interaction. In this case the signal polariton is stimulated by the probe at $k=0$, due to bosonic behavior. In figure 2.5, experimental data from Savidis et al [16] is presented. The probe amplification increases exponentially with growing pump intensity, representing a strong evidence of stimulated amplification. Once again, the idler spot is less intense than the probe one. This fact is a clear sign of the different polariton radiative decay in various momentum states. Moreover, the coupling with the semiconductor environment through the excitonic component of the polariton is responsible for this assimetry and the clear difference in signal and idler emission patterns (see Figures 2.4 and $2.5)$. 

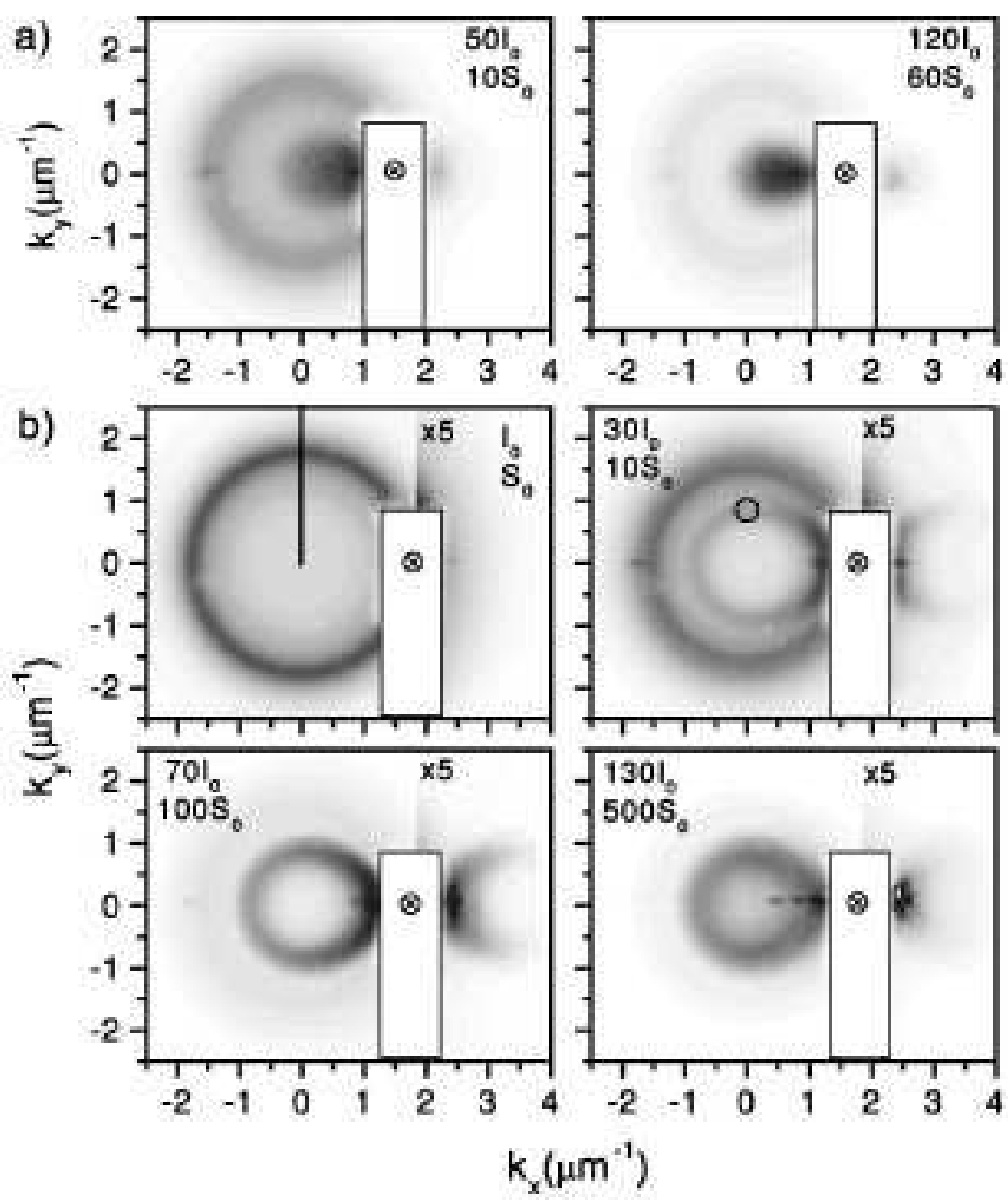

Figure 2.4: Experimental data from Langbein [2]. Emission for a resonant pulsed excitation at (a) $k_{p}=(1.5,0) \mu m^{-1}$ and (b) $k_{p}=(1.73,0) \mu m^{-1}$. 


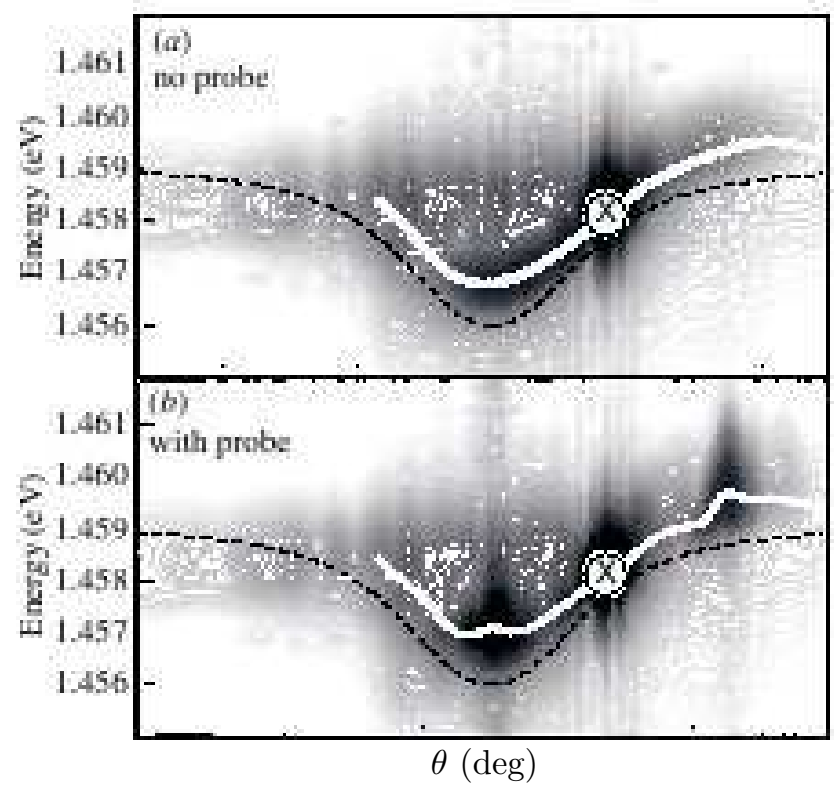

Figure 2.5: Experimental data from Savidis et al [16]. (a) Contour plot of the lower polariton emission as a function of the detection angle and energy. The resonantly pump beam is indicated by the cross. (b) Emission patter with a probe beam at $k=0$.

\subsection{Coupling with the semiconductor environment}

In the previous discussion it became clear that decoherence induced by the coupling with the semiconductor environment is a rather important aspect in the polaritons dynamics, specially in high momentum states. This type of coupling is modeled by phonons, which are bosons as well. Through its underlying exciton component, polaritons interact with phonons leading into a relaxation dynamics process. The relaxation times are very short for photonlike polaritons and much longer for excitonlike polaritons $[18,20]$. The theoretical description of such effects by this authors rely on the transition rates for the rate equation which are calculated from the underlying microscopic many-body model with the quantum mechanical "golden rule".

In our model, we will work with the full Hamiltonian, meaning polariton-polariton and polaritonphonon interactions. So far we have reviewed the polariton-polariton interaction Hamiltonian (2.12), and in this section we will work the usual exciton and phonon interaction Hamiltonian to obtain an expression for this decoherent scattering process in terms of the polariton basis.

The interaction Hamiltonian between bosonic excitons and acoustic phonons is in the long wavelength limit $[21,22,23]$ 


$$
H_{P H}=\frac{1}{\sqrt{V}} \sum_{k, q} g_{q} b_{k+q}^{\dagger} b_{k}\left(c_{q}+c_{-q}^{\dagger}\right)
$$

with

$$
g_{q}=G \sqrt{\hbar \omega_{q}}
$$

The coupling constant $G$ is related to the material parameters by $G^{2}=D^{2} / \rho s^{2}$, where $D$ is the band gap deformation potential constant, $\rho$ is the crystal density, and $s$ the sound velocity. The phonon anihilation operators are $c_{q}$ with bosonic conmutation rules $\left[c_{q}, c_{q^{\prime}}^{\dagger}\right]=\delta_{q, q^{\prime}}$. The crystal volume is $V$ with periodic boundary conditions. In order to transfrom the exciton-phonon interaction into an effective polariton-phonon coupling we need only to replace the exciton operators by their respective upper and lower polariton operators. Once again, we dropped the upper polariton operators and we are left with

$$
H_{P H}=\sum_{k, q} V_{X P} p_{k+q}^{\dagger} p_{k}\left(c_{q}+c_{-q}^{\dagger}\right)
$$

where the coupling magnitude

$$
V_{X P}=\frac{1}{\sqrt{V}}\left|X_{k+q}\right| g_{k, q}
$$

is only dependant on the excitonic component as expected.

With this transformation we have completed all the neccesary framework to study polariton dynamics. For completness, the total Hamiltonian of interacing polaritons coupled with the environment is

$$
H=\sum_{k} E(k) p_{k}^{\dagger} p_{k}+\sum_{q} e(q) c_{q}^{\dagger} c_{q}+H_{P P}+H_{P H}
$$

where the first two terms correspond to the free energies of the polartions and phonons respectively. The third term is the polariton-polarition interaction Hamiltonian (2.12) and the last term is the coupling with the semiconductor environment (2.16). The last two terms of (2.18) makes this 
Hamiltonian highly nonlinear. Hence, most theoretical descriptions on polaritons dynamics require some kind of approximation procedure, as mention in the beggining. In the next chapter we will introduce a new stochastic scheme that will prove to be an ideal tool in the study of interacting open systems. A first straightforward application will be performed for microcavity polaritons in chapter 4 . 


\section{Chapter 3}

\section{Exact dynamics of interacting systems: Stochastic approach}

The mathematical treatment of the many-body problem of a system composed by interacting bosons coupled to a reservoir (which is modelled by phonons, bosons as well), is quite complicated due to the high dimensionality of the Hilbert space and the nonlinearities introduced by the interactions. Mean-field and other approximation theories are commonly used to discuss the dynamics and the stationary behavior of the system mentioned above. Unfortunately, in systems such as the one we are interested (see chapter 2), which a behavior governed completely by the strong coupling with the reservoir, and long range interactions between the excitonic part of the polaritons, these approximated theories fail to describe correctly the observed behavior. This fact leaves an open door for new theories that allow us to better understand the basic properties of these interesting systems.

In this chapter we present a stochastic reformulation of the problem at hand and show how, with the help of these new theoretical tool, we are in a position to discuss the microcavity polariton system. Our main concern will be to prove that the stochastic solution does not only go further than mean-field theories but that it is also in principle exact.

The presentation of the method is as follows. We present the general description of two stochastic schemes, each one describing a different set of problems. In the first one, the evolution of a closed system of interacting bosons is analyzed in terms of the stochastic evolution of a single particle state [7]. In the second scheme [10,11], the coupling between a system and its surroundings is modelled by splitting the whole state vector of the system-environment in pairs of stochastic wave equations. Using these ideas and the same line of procedure, we developed a new stochastic method for the general problem of an open interacting boson system coupled with an environment. 
With this approach, the complete dynamics are transformed into a simple scheme. The bosons are represented by the stochastic evolution of a single particle state and the environment is decoupled into a wave function stochastic equation. When averaged over a great number of realizations, this scheme unravels the complete exact dynamics.

\subsection{N-Boson system with two-body interaction. Closed sys- tem}

A particular problem that has been recently studied with the help of stochastic equations of motion is a system of $\mathrm{N}$ interacting bosons in a specific confinement. The interest in this system lies in what has become a main subject of research for the atomic physics community since the first experimental realization of atomic Bose-Einstein condensates a few years ago [26].

In this case, perturbative and other approximation theories have been successful in the description of the steady state and the dynamics in the limit of weak interactions. Carrusotto et al have recently developed a method [7] with the help of stochastic wave functions to fully include long range and strong interaction between particles, leading a step closer in the understanding of the role of atomic interactions in the Bose-Einstein condensation (BEC). Our main aim in this section is to present the general description of this method and include some examples that will be of key importance for the understanding of the new stochastic scheme we will introduce below.

\subsubsection{Stochastic formulation of a N-Boson closed system}

The Hamiltonian of the interacting bosons trapped in a confinement potential in terms of the Bose field operators $\hat{\Psi}(x)$ can be written as

$$
H=\int d x \hat{\Psi}^{\dagger}(x) h_{0} \hat{\Psi}(x)+\frac{1}{2} \iint d x d x^{\prime} \hat{\Psi}^{\dagger}(x) \hat{\Psi}^{\dagger}\left(x^{\prime}\right) V\left(x-x^{\prime}\right) \hat{\Psi}\left(x^{\prime}\right) \hat{\Psi}(x)
$$

where $h_{0}=-\left(\hbar^{2} / 2 m\right) \nabla^{2}+V_{\text {ext }}(x)$ with $V_{\text {ext }}(x)$ an external potential of confinement. The interactions are via a two-body potential $V\left(x-x^{\prime}\right)$. Let us assume that at any given time, all the bosons are in the same single particle state $\phi$, then the total state vector is

$$
|\Phi\rangle=|N: \phi\rangle
$$

A general proof of the over-completness of the Hartree-Fock states is done in [27]. In other words any state vector can be expanded onto a set of Hartree-Fock states as in (3.2). Under the assumption 
(3.2) the density matrix is $\rho=|N: \phi\rangle\langle N: \phi|$. Then the only task remaining would be to find an equation of motion for the single particle state $\phi(x)$.

This can be easily done by solving the Schrödinger equation $(\hbar=1)$

$$
i \frac{d}{d t} \psi=\hat{H} \psi
$$

(operate the Hamiltonian (3.1) to the state vector (3.2)) or the von Neumann equation ${ }^{1}(3.30)$ (calculate the commutator of the Hamiltonian and the density matrix).

Let us first separate the action of the bosonic operators in the longitudinal and orthogonal contribution to the single particle state, leaving us with

$$
\hat{\Psi}(x)|N: \phi\rangle=\sqrt{N} \phi(x)|N-1: \phi\rangle
$$

and

$$
\hat{\Psi}^{\dagger}(x)|N: \phi\rangle=\sqrt{N+1} \frac{\phi^{*}(x)}{\|\phi\|^{2}}|N-1: \phi\rangle+\hat{\Psi}_{\perp}^{\dagger}(x)|N: \phi\rangle .
$$

Finally, calculating explicitly the right hand side of the Schrödinger equation (3.3) for the HartreeFock state, using the previous definitions and collecting the terms containing $\hat{\Psi}_{\perp}^{\dagger}(x)$ one obtains

$$
\begin{array}{r}
H|N: \phi\rangle=\sqrt{N}\left(\int d x G(x) \hat{\Psi}^{\dagger}(x)\right)|N-1: \phi\rangle \\
+\frac{\sqrt{N(N-1)}}{2}\left(\iint d x d x^{\prime} V\left(x-x^{\prime}\right) \phi(x) \phi\left(x^{\prime}\right) \hat{\Psi}_{\perp}^{\dagger}(x) \hat{\Psi}_{\perp}^{\dagger}\left(x^{\prime}\right)\right)|N-2: \phi\rangle
\end{array}
$$

where we have defined

$$
G(x)=-i\left[h_{0}+\frac{(N-1)}{\|\phi\|^{2}} \int d x^{\prime} V\left(x-x^{\prime}\right)\left|\phi\left(x^{\prime}\right)\right|^{2}-\frac{N-1}{2} \frac{\langle\phi \phi|V| \phi \phi\rangle}{\|\phi\|^{4}}\right] \phi(x) .
$$

On the left hand side of equation (3.3), for a sufficient small increment in time $d t$, the single particle state changes in an amount $d \phi$, leaving the total state of the system as $|N: \phi+d \phi\rangle$ which can be written as

\footnotetext{
${ }^{1}$ The formal proof presented by Carusotto et al. is with the total density matrix in [??]. We will only do it in a shorter fashion with the Schrödinger equation
} 


$$
|N: \phi+d \phi\rangle=\frac{\left(\hat{a}_{\phi}^{\dagger}+\hat{a}_{d \phi}^{\dagger}\right)^{N}}{\sqrt{(} N !)}|0\rangle .
$$

Using the binomial expansion up to second order terms in $d \phi$

$$
|N: \phi+d \phi\rangle=\frac{1}{\sqrt{N !}}\left[\left(\hat{a}_{\phi}^{\dagger}\right)^{N}+N\left(\hat{a}_{\phi}^{\dagger}\right)^{N-1} \hat{a}_{d \phi}^{\dagger}+\frac{N(N-1)}{2}\left(\hat{a}_{\phi}^{\dagger}\right)^{N-2}\left(\hat{a}_{d \phi}^{\dagger}\right)^{2}\right]|0\rangle
$$

Rewritting the last expression in terms of the Boson field operators and using the known basis transformation $\hat{a}_{\phi}=\int d x \phi^{*}(x) \hat{\Psi}(x)$ we are left with the following variation of the total state vector $(3.2)$

$$
\begin{array}{r}
d|N: \phi\rangle=\sqrt{N}\left(\int d x d \phi(x) \hat{\Psi}^{\dagger}(x)\right)|N-1: \phi\rangle \\
+\frac{\sqrt{N(N-1)}}{2}\left(\iint d x d x^{\prime} d \phi(x) d \phi\left(x^{\prime}\right) \hat{\Psi}^{\dagger}(x) \hat{\Psi}^{\dagger}\left(x^{\prime}\right)\right)|N-2: \phi\rangle .
\end{array}
$$

If we consider $d \phi$ to be proportional to the time step, the last term in (3.10) is neglected and the second term in (3.6) cannot be accounted for, this is known as the Hartree-Fock mean field approximation. Here, the single particle state follows the deterministic evolution governed by

$$
d \phi(x)=-i d t G(x)
$$

To completely recover the evolution of the bosonic system under the hamiltonian (3.1), one would need to find an evolution of $\phi$ such that (3.10) and (3.6) satisfy the Schrödinger equation. In order to do so, a stochastic term proportional to $\sqrt{d t}$ is included in (3.11)

$$
d \phi(x)=-i d t G(x)+d M(x) .
$$

The properties of this stochastic term are straightforward since one needs to fulfil the condition

$$
E[d|N: \phi\rangle]=-i d t H|N: \phi\rangle,
$$

Then, it is easy to verify that $d M$ must have zero mean and a correlation function defined by 


$$
E\left[d M(x) d M\left(x^{\prime}\right)\right]=\frac{d t}{i \hbar} \mathcal{Q}_{x} \mathcal{Q}_{x^{\prime}}\left[V\left(x-x^{\prime}\right) \phi(x) \phi\left(x^{\prime}\right)\right]
$$

where $\mathcal{Q}_{x}$ projects to the space orthogonal to $\phi$. In other words, the mean-field approximation takes into account the projection of the Hamiltonian (3.1) in to the longitudinal subspace of the single particle state, while the stochastic term recovers the projection into the orthogonal subspace, last term in (3.6).

Finally, to obtain the formally exact density matrix as the stochastic average over multiple realizations, we impose that each sample has to be taken as the dyadics $\rho=\left|N: \phi_{1}\right\rangle\left\langle N: \phi_{2}\right|$, where $\phi_{1,2}$ each follow independent stochastic trajectories [7, 24].

\subsubsection{Evolution of the single particle state}

In principle this complex problem where the $\mathrm{N}$ particles are interacting with each other, and the symmetrization of the total state vector living in the N-particle subspace has to be performed, turns into a rather simple task. One should only worry about the evolution of a single particle state. If there were no particle-particle interactions this is trivial, since every boson behaves independently, but with the help of Mean Field approach and a stochastic term the evolution of interacting bosons can be studied in a similar manner.

For $\mathrm{N}$ bosons that have $\mathrm{M}$ possible states available, the total state vector describing the system lives in a subspace of symmetric wave functions of the total Hilbert space of dimension $M^{N}$. Since the total state vector can also be expressed as placing the $\mathrm{N}$ boson in the same single particle state (where the single particle state is any state from the Hilbert space of a single particle), the method proposes that it is necessary only to follow the evolution of the single particle state in order to recover the complete evolution. A graphical description of this idea is presented in figure (3.1).

Another important aspect of this method is the calculation of the observables. The density matrix is the average over the $R$ trajectories of the Hartree-Fock states

$$
\rho(t)=\frac{1}{R} \sum_{i=1}^{R}\left|N: \phi_{1}^{(i)}(t)\right\rangle\left\langle N: \phi_{2}^{(i)}(t)\right|,
$$

and the expectation value of an operator $\hat{O}$ is given by

$$
\langle\hat{O}\rangle=\frac{1}{R} \sum_{i=1}^{R}\left\langle N: \phi_{2}^{(i)}(t)|\hat{O}| N: \phi_{1}^{(i)}(t)\right\rangle .
$$




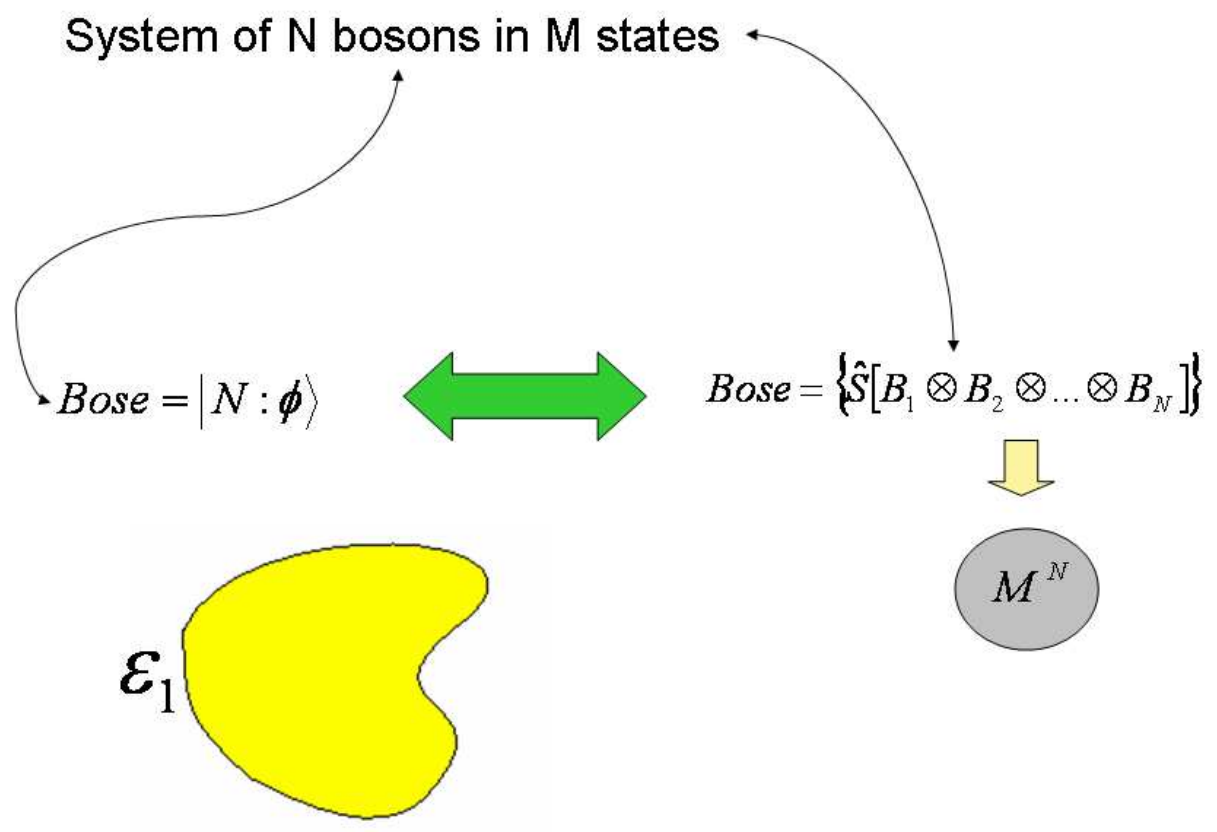

Figure 3.1: In the case of $\mathrm{N}$ bosons in $\mathrm{M}$ possible states, a vector that belongs in the subspace of symmetric functions of the total Hilbert space can be expanded in a Hartree-Fock state, where the $\mathrm{N}$ particles are placed in the same single particle state. The last could be any vector in the single particle Hilbert space. 


\subsubsection{Real time evolution}

We first consider a simple model where the dynamics is revised. The model consist of two coupled condensates for which the number of bosons is conserved. Two particle contact interactions are allowed inside each condensate. The hamiltonian of this system is

$$
H=\frac{\Omega}{2}\left(a_{0}^{\dagger} a_{1}+a_{1}^{\dagger} a_{0}\right)+\kappa\left(a_{1}^{\dagger 2} a_{1}^{2}+a_{0}^{\dagger 2} a_{0}^{2}\right)
$$

where $a_{i}\left(a_{i}^{\dagger}\right)$ annihilates (creates) a boson in the mode $\mathrm{i}$, with $i=0,1$. The strength of the coupling between the modes is $\Omega$ and the strength of the particle interactions is $\kappa$. The total state vector for the $\mathrm{N}$ bosons in the two modes is

$$
|\psi(t)\rangle=\sum_{k=0}^{N} c_{k}(t)|k, N-k\rangle .
$$

In this number of particle basis, $c_{k}(t)$ is the amplitude of a state with $k$ bosons in mode $u_{0}(x)$ and $N-$ $k$ in the mode $u_{1}(x)$. Due to the quadratic terms in the hamiltonian, this system has no analytical solution, but direct numerical integration is possible. This is done by solving the Schrödinger equation (3.3), in particular we need to solve the differential equations for the amplitudes

$$
\begin{array}{r}
\frac{d c_{k}(t)}{d t}=-\frac{i}{2}\left[c_{k-1}(t) \sqrt{(N-k+1) k}+c_{k+1}(t) \sqrt{(k+1)(N-k)}\right] \\
-i \kappa c_{k}(t)[k(k-1)+(N-k)(N-k-1)]
\end{array}
$$

This way, the evolution of the system is found by solving the $N+1$ coupled differential equations. We are interested in calculating the probability of occupation of the mode $u_{0}(x)$, which is defined as $p(t)=\frac{1}{N} \sum_{k=0}^{N}\left|c_{k}(t)\right|^{2} k$.

On the other hand, the stochastic solution is as follows. The single particle vector can be expressed in terms of the two modes

$$
\phi_{\nu}(x)=\phi_{0 \nu} u_{0}(x)+\phi_{1 \nu} u_{1}(x)
$$

and the action of the field operator can also be split as

$$
\hat{\Psi}(x)=a_{0} u_{0}(x)+a_{1} u_{1}(x) .
$$


With the system's hamiltonian (3.17) we find the corresponding Mean-Field evolution (3.7)

$$
\begin{array}{r}
G(x)=\frac{\hbar \Omega}{2}\left[\phi_{1 \nu} u_{0}(x)+\phi_{0 \nu} u_{1}(x)\right]+\hbar \kappa(N-1)\left[\frac{\left|\phi_{0 \nu}\right|^{4}+\left|\phi_{1}\right|^{4}}{\left(\left|\phi_{0 \nu}\right|^{2}+\left|\phi_{1 \nu}\right|^{2}\right)^{2}}\right]\left[\phi_{0 \nu} u_{0}(x)+\phi_{1 \nu} u_{1}(x)\right] \\
+\frac{2(N-1)}{\left|\phi_{0 \nu}\right|^{2}+\left|\phi_{1 \ni}\right|^{2}}\left[\left|\phi_{0 \nu}\right|^{2} u_{0}(x)+\left|\phi_{1 \nu}\right|^{2} u_{1}(x)\right] .
\end{array}
$$

Introducing this expression in the single particle state evolution (3.11) and collecting terms for $u_{0}(x)$ and $u_{1}(x)$, one is left with the set of stochastic equations for $\phi_{0 \nu}$ and $\phi_{1 \nu}$

$$
\begin{array}{r}
\phi_{0 \nu}(t+d t)=\phi_{0 \nu}(t) \\
-i d t\left[\frac{\Omega \phi_{1 \nu}}{2}+\frac{2 \kappa(N-1)}{\left\|\phi_{\nu}\right\|^{2}}\left|\phi_{0 \nu}\right|^{2} \phi_{0 \nu}-\frac{\kappa(N-1)}{\left\|\phi_{\nu}\right\|^{4}}\left(\left|\phi_{0 \nu}\right|^{4}+\left|\phi_{1 \nu}\right|^{4}\right) \phi_{0 \nu}\right]+d M_{0 \nu} \\
\phi_{1 \nu}(t+d t)=\phi_{1 \nu}(t) \\
-i d t\left[\frac{\Omega \phi_{0 \nu}}{2}+\frac{2 \kappa(N-1)}{\left\|\phi_{\nu}\right\|^{2}}\left|\phi_{1 \nu}\right|^{2} \phi_{1 \nu}-\frac{\kappa(N-1)}{\left\|\phi_{\nu}\right\|^{4}}\left(\left|\phi_{0 \nu}\right|^{4}+\left|\phi_{1 \nu}\right|^{4}\right) \phi_{1 \nu}\right]+d M_{1 \nu}
\end{array}
$$

As it was shown before the total state vector for a particular time is given by the average over the stochastic trajectories $|\psi(t)\rangle=\langle\mid N: \phi\rangle\rangle$. In this sense, the two coupled stochastic equations (3.23) and (3.24) give the full dynamics of the system independently of the number of particles ${ }^{2}$. For this simple example, the full advantage of the method becomes evident. This is a trade off between the evolution of a symmetric vector in the $\mathrm{N}$ particle subspace and a large number of simulations for a vector in the single particle space.

In order to calculate the occupation probability of $u_{0}(x)$ in terms of $\phi_{0,1}$ we need to rewrite (3.16) for $\hat{O}=a_{0}^{\dagger} a_{0} / N$. This is

$$
\begin{array}{r}
p_{0}(t)=\frac{1}{N} \frac{1}{R} \sum_{i=1}^{R}\left\langle N: \phi_{2}^{(i)}(t)\left|\hat{a}_{0}^{\dagger} a_{0}\right| N: \phi_{1}^{(i)}(t)\right\rangle \\
p_{0}(t)=\frac{1}{R} \sum_{i=1}^{R} \phi_{02}^{*(i)}(t) \phi_{01}^{(i)}(t)\left\langle N-1: \phi_{2}^{(i)}(t) \mid N-1: \phi_{1}^{(i)}(t)\right\rangle \\
p_{0}(t)=\frac{1}{R} \sum_{i=1}^{R} \phi_{02}^{*(i)}(t) \phi_{01}^{(i)}(t)\left[\phi_{2}^{*(i)}(t) \phi_{1}^{(i)}(t)\right]^{N-1} \\
p_{0}(t)=E\left\{\phi_{02}^{*}(t) \phi_{01}(t)\left[\phi_{2}^{*}(t) \phi_{1}(t)\right]^{N-1}\right\} .
\end{array}
$$

\footnotetext{
${ }^{2}$ The number of particles influences the stochastic evolution by forcing higher number of simulations to reach convergence, since the effective interaction is greater and the solution diverges from Mean-Field
} 


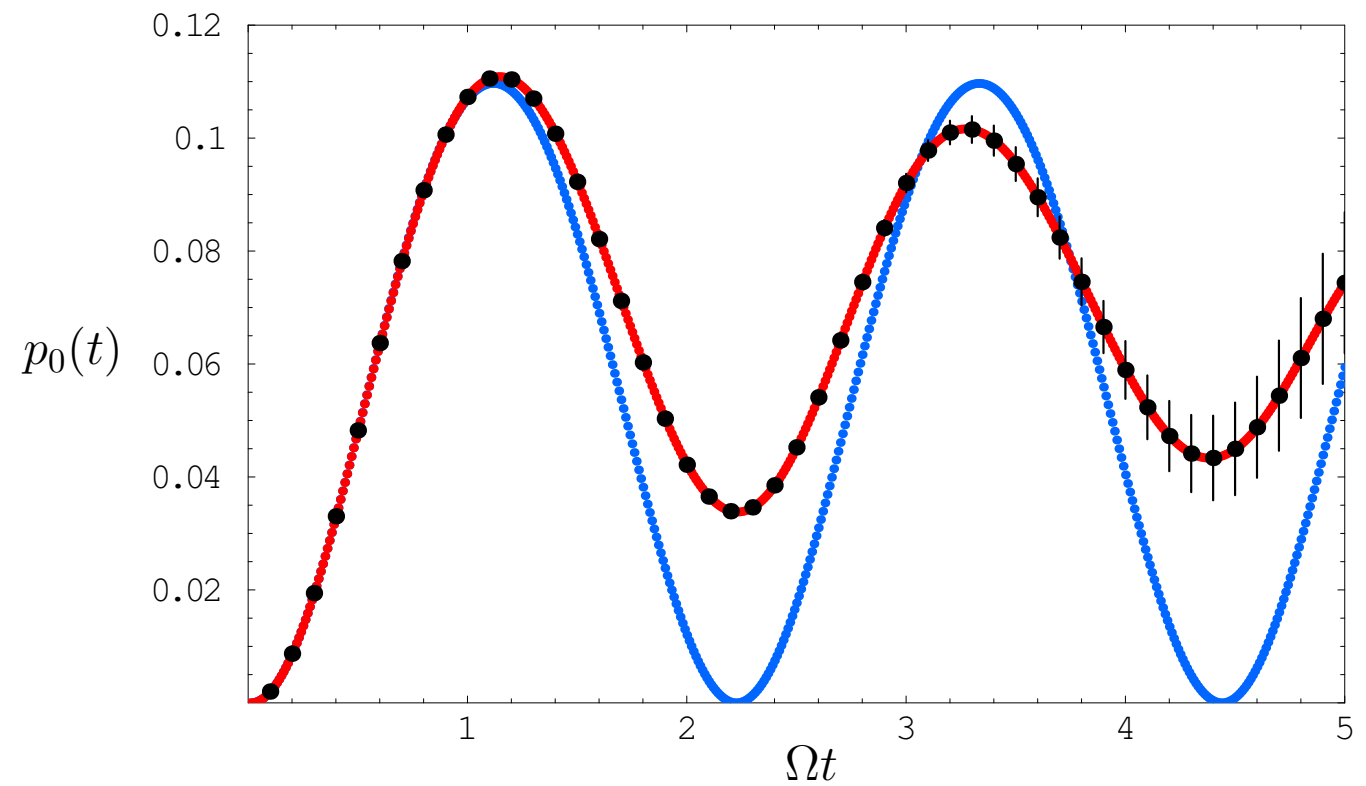

Figure 3.2: Occupation probability of mode $u_{0}$. The results are shown for $N=17$ particles and $u_{0}$ initially empty. The interaction strength was chosen as $\kappa=0.1 \Omega$. The stochastic solution (black) which is shown with its respective error bars recovers the exact results (red) for this particular range of time. This is done with $10^{6}$ realizations and a time step of $d t=0.01 \Omega$. The mean-field solution (blue) exhibits perfect Rabi-like oscillations being unable to reproduce the real damped oscillatory behavior.

In figure (3.2) the occupation probability for the mode $u_{0}(x)$ is displayed, for $N=17$ and all the particles initially in mode $u_{1}(x)$. We show the results obtained by direct numerical integration (3.19), by standard mean-field theory ${ }^{3}$ and with the stochastic method. A first important result to remark is the excellent convergence exhibited by the solution. Also, the collapse and revivals captured in the dynamics cannot be described by mean-field approximations which only show perfect Rabi-like oscillations; the first one is a pure quantum property.

The method is definitely a very practical tool for systems where approximation theories fail to give accurate predictions, specially for the quantum behavior. This is the case when the interest lies in the study of high order coherence and other quantum properties.

Even though this was presented as a simple model, it gives a starting point for the general polariton system in which we are interested. We will see, that with the proper extension this is a common model for polariton dynamics (two level) [6].

\footnotetext{
${ }^{3}$ In this case, since the stochastic evolution is performed on top of a mean-field dynamics, it is only necessary to make the stochastic terms equal to zero.
} 


\subsubsection{Imaginary time evolution: Thermal equilibrium}

Another result of the stochastic method developed by Carusotto et al, is the possibility of studying the statistical properties of interacting bosonic systems in thermal equilibrium. This particular extension of the method will not be used below in the polariton problem, but it is, from the failure to extend the method to combine thermal relaxation and strong particle interactions, that there exist a necessity to develop a brand new approach (a detailed discussion will be done in Section $3.3)$.

The system of interest consists of $\mathrm{N}$ bosons in a one dimensional harmonic confinement. The interactions are assumed to be of contact in the position space $V\left(x-x^{\prime}\right)=g \delta\left(x-x^{\prime}\right)$ with $g$ as the interaction strength. The hamiltonian (3.1) turns into

$$
H=\int d x \hat{\Psi}^{\dagger}(x) h_{0} \hat{\Psi}(x)+\frac{g}{2} \int d x \hat{\Psi}^{\dagger}(x) \hat{\Psi}^{\dagger}(x) \hat{\Psi}(x) \hat{\Psi}(x)
$$

with $h_{0}=-\left(\hbar^{2} / 2 m\right) \nabla^{2}+\frac{1}{2} m \omega^{2} x^{2}$. Using the transformation $i d t \rightarrow \frac{d \tau}{2}$, where $\tau$ is associated with the inverse of the temperature $\left(\tau=1 / K_{B} T\right)$, the Liouville equation becomes

$$
d \rho(\tau)=-\frac{d \tau}{2}[H, \rho(\tau)]
$$

The solution of equation (3.27) for a given $\tau$ is the density matrix at thermal equilibrium. This is known as the imaginary time evolution. In the not norm conserving evolution, the initial "time" is associated with $T \rightarrow \infty$, and the density matrix is $\rho(0)=1_{N}$. After an infinite "time" the temperature is zero.

With this transformation and using the hamiltonian (3.26), the evolution of the single particle state is

$$
\begin{aligned}
d \phi_{\nu}(x) & =-\frac{d \tau}{2}\left[\frac{p^{2}}{2 m}+\frac{1}{2} m \omega^{2} x^{2}+g(N-1) \frac{\left|\phi_{\nu}(x)\right|^{2}}{\left\|\phi_{\nu}\right\|^{2}}\right. \\
& \left.-\frac{g(N-1)}{2} \frac{\int d x^{\prime}\left|\phi_{\nu}\left(x^{\prime}\right)\right|^{4}}{\left\|\phi_{\nu}\right\|^{4}}\right] \phi_{\nu}(x)+d M_{\nu}(x) .
\end{aligned}
$$

The statistical properties of the confined bosons at temperature $T$ is calculated from the density matrix conformed by the average of the Hartree-Fock state trajectories. This procedure is similar to the one presented before, and the calculation of the observables is the same. The additional task 

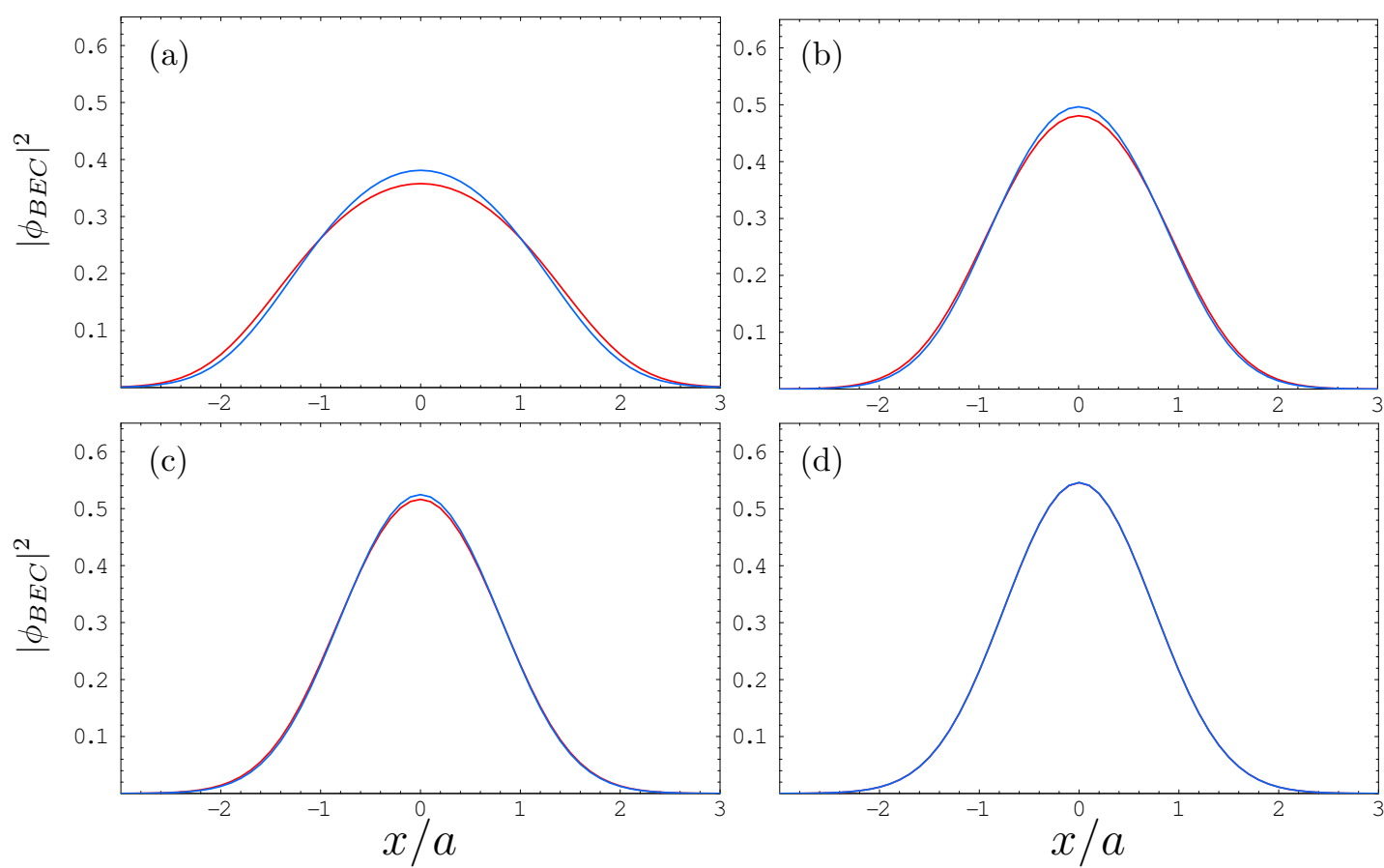

Figure 3.3: Condensate wave function density profile for $N=151$ particles in an harmonic confinement at different temperatures $k_{B} T / \hbar \omega=1$ (a), 10 (b), 20 (c) and 50 (d). The interaction strength is $g=0.1 \hbar \omega$, and the units are scaled to the proper length of the harmonic oscillator $a=\sqrt{\hbar / m \omega}$. Predictions of the stochastic method (red) are compared with the Bogoliubov mean-field approximation (blue). The results are obtain for $10^{4}$ trajectories.

is to determine the initial sample of the infinite density operator. Details are discussed in [24]. Here we present the condensate wave function density. Since the system consists of interacting particles this corresponds to the wave function of dominant eigenvalue in the single particle density matrix (this criteria is commonly used to test BEC).

In figure (3.3) the condensate wave function density is shown for $N=151, g=0.1 \hbar \omega$ and different temperatures. The results are compared with Bogoliubov mean-filed approximations. At high temperatures the thermal behavior is dominant and the interactions play a less important role than in the low temperature regime. Is in the last case where the predictions of Mean-Field and the stochastic exact approach become evidently different.

This type of system is non solvable and results from the stochastic scheme can only be compare with Bogoliubov predictions for weak interactions. However, for the particular case $N=2$, we showed in a previous work [28] that in the strong interaction regime the bosons exhibit a quasi-fermionic behavior [29]. Particulary, the single particle wave function of the condensed was antisymmetric and the probabilty of finding a particle in the center of the confinment was minimum $(T=0)$. This results were in the framework of an analytical solvable model. With the stochastic scheme we 


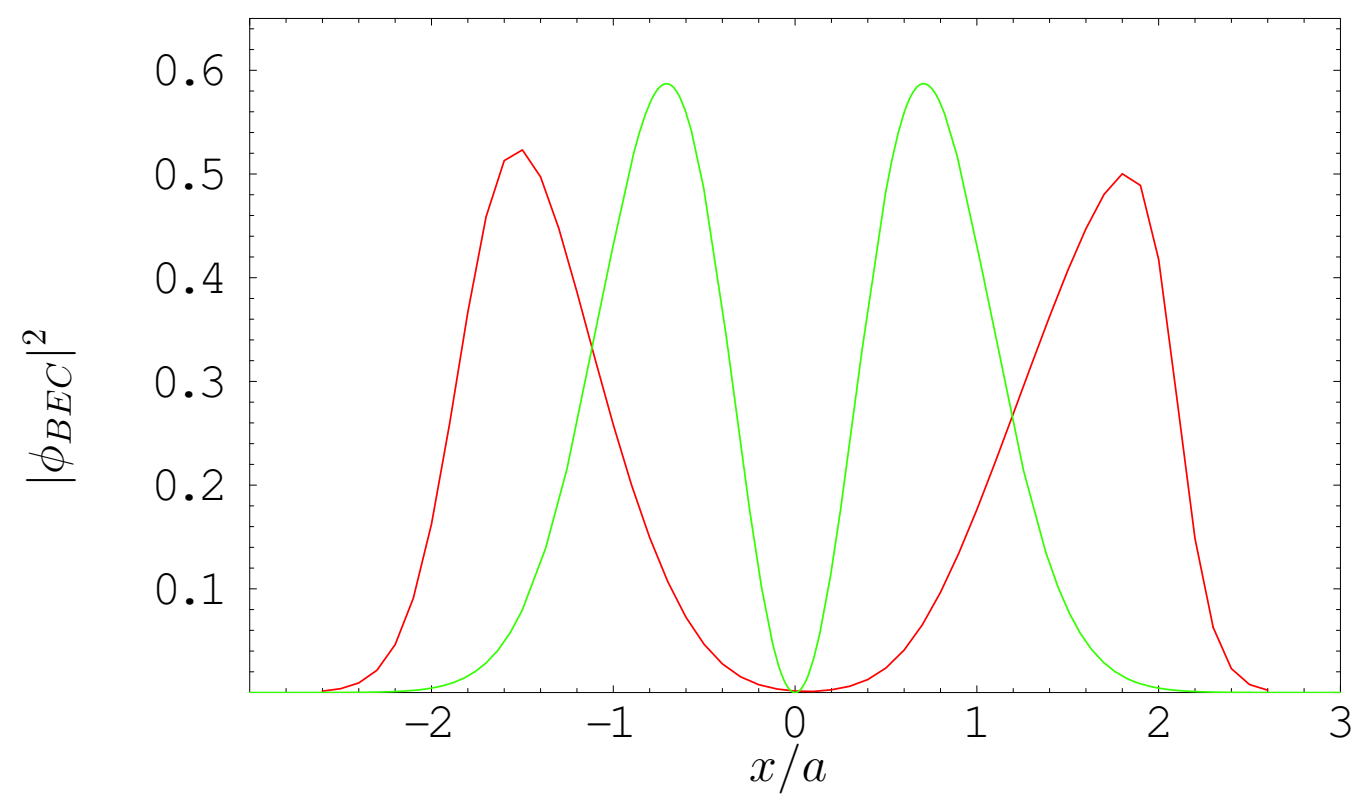

Figure 3.4: Condensate wave function density profile for $N=2$ bosons in an harmonic confinement at temperature $T=0$. The interaction strength is $g=100 \hbar \omega$ for the stochastic results (red), and $g \rightarrow \infty$ for the exact (green) results (see Reference [28]). The units are scaled to the proper length of the harmonic oscillator $a=\sqrt{\hbar / m \omega}$. The results are obtain for $10^{5}$ trajectories.

calculate the condensate wave function density for $N=2$ and $g=100 \hbar \omega$ (see Figure 3.4). Even though there is a discrepancy between the exact and the stochastic curves, provided by the fact that stochastic results were calculated for a large but finite interaction strength, the real behavior is well captured. While Bogoliubov predictions fail dramatically for large interactions strengths (not shown).

\subsection{Exact formulation for open quantum systems}

The description of open quantum systems with Stochastic Schrödinger Equation (SSE) has been a growing field in recent years. This is due to the possibility of transforming the density matrix evolution into a stochastic evolution of wave-packets. Quantum jump approaches have been developed with great success, and the dissipative effects captured in the low coupling regime. In systems such as microcavity polaritons, where the semiconductor environment induces decoherence, the non-Markovian dynamics plays an important role. More recently, a Monte Carlo wave function method was developed by Breuer [10], which enables the study of the complete behavior of open quantum systems, even in the strong coupling regime.

The basic idea of the method, is to evolve a pair of state vectors of the total system, leading to 
density matrices of the type $\rho=\left|\Phi_{1}\right\rangle\left\langle\Phi_{2}\right|$. The total state vector is assumed to be at all times the direct product of the open system and the environment vector as $\left|\Phi_{\nu}\right\rangle=\psi_{\nu} \otimes \chi_{\nu}$ (for $\nu=1,2$ ). Each vector follows independent stochastic trajectories that in average reproduces the complete dynamics.

Our main goal is to present this formulation in terms of diffusive wave-function processes using pairs of wave-packets. We will also show a couple of examples which put in evidence the accuracy of the method.

\subsubsection{Diffusive stochastic wave function approach}

An open quantum system can be described by a system coupled with its environment. The Hamiltonian describing this complex system, in the interaction picture, is given by

$$
H_{I}=\sum_{\alpha} A_{\alpha}(t) \otimes B_{\alpha}(t) .
$$

The $A_{\alpha}(t)$ and $B_{\alpha}(t)$ are interaction picture operators acting on the system and environment respectively. As it should be clear later, the most general state for this problem has to be a superposition of individual states of the system and environment. In this particular problem the dimensionality of the total Hilbert space is $H=H_{S} \otimes H_{E}$. Any attempt to study the dynamics of the system is restricted to solving the von Neumann equation $(\hbar=1)$

$$
\frac{d}{d t} \rho(t)=-i\left[H_{I}(t), \rho(t)\right]
$$

and tracing over the environment $\rho_{S}(t)=\operatorname{Tr}_{E}(\rho(t))$.

In the stochastic method we assume that the state vector can be written as the direct product of the states of the system and the environment $[10,11]$

$$
\left|\Phi_{\nu}(t)\right\rangle=\psi_{\nu}(t) \otimes \chi_{\nu}(t)
$$

with $\psi_{\nu}(t)$ the state vector of the system and $\chi_{\nu}(t)$ the state vector of the environment. Using (3.31) the ansatz for the density matrix becomes

$$
R(t)=\left|\Phi_{1}(t)\right\rangle\left\langle\Phi_{2}(t)\right| .
$$


Here the ket and bra follow different and independent evolutions.

Finally, the evolution of the whole system under Hamiltonian (3.29) is replaced by the set of Stochastic Schrödinger Diferential Equations (SSDE)

$$
d \psi_{\nu}(t)=\frac{-i d t}{\hbar} \sum_{\alpha} d W_{\alpha \nu}(t) A_{\nu} \psi_{\nu}(t)
$$

and

$$
d \chi_{\nu}(t)=\sum_{\alpha} d W_{\alpha \nu}(t) B_{\nu} \chi_{\nu}(t)
$$

where the system is decoupled from its environmnet. Here $d W_{\alpha \nu}$ are stochastic Gaussian variables under the conditions

$$
E\left[d W_{\alpha \nu}(t) d W_{\alpha^{\prime} \nu^{\prime}}(t)\right]=\delta_{\alpha \alpha^{\prime}} \delta_{\nu \nu^{\prime}} .
$$

and $E\left[d W_{\alpha \nu}\right]=0$.

It should not be inferred from the latter that the stochastic evolution $(3.33,3.34)$ is unique. The condition that needs to be imposed in the evolution is to reproduce in average the von Neumman equation (3.30), more specifically

$$
d \rho(t)=E[d R(t)]=E\left[\frac{-i d t}{\hbar}\left[H_{I}, R(t)\right] .\right.
$$

In fact, the ability to generate simple schemes that meet the condition (3.36) will enable us not only to optimize our stochastic trajectories, but to readjust the method to suite for other problems of interest. In the following section we will demonstrate how this simple scheme can in fact reproduce the exact solution for any given system and environment.

\subsubsection{Exact and stochastic dynamics}

Up to this point we have presented the problem of interest and we even introduced a possible set of solutions. What is left to be done is to obtain a stochastic equation of motion for the random 
variable $\mathrm{R}(\mathrm{t})$ defined in (3.31), and then perform the expectation value to verify condition (3.36). Employing the calculus of stochastic equations one finds that a small increment in $R(t)$ is

$$
d R=\left|d \Phi_{1}\right\rangle\left\langle\Phi_{2}|+| \Phi_{1}\right\rangle\left\langle d \Phi_{2}|+| d \Phi_{1}\right\rangle\left\langle d \Phi_{2}\right|
$$

The third term in (3.37) involves products of $d W_{\alpha 1} d W_{\alpha^{\prime} 2}$ and by virtue of condition (3.35) they vanish. We are left to find the increments of the state vector $\left|d \Phi_{1}\right\rangle$ and $\left\langle d \Phi_{2}\right|$. With the separability that was imposed in the total state vector (3.31) any increment can be written as

$$
\left|d \Phi_{\nu}\right\rangle=d \psi_{\nu} \otimes \chi_{\nu}+\psi_{\nu} \otimes d \chi_{\nu}+d \psi_{\nu} \otimes d \chi_{\nu}
$$

By using the equations of motion for the system (3.33) and environment (3.34), a small increase in $|\Phi\rangle$ becomes

$$
\begin{aligned}
\left|d \Phi_{\nu}\right\rangle=-\frac{i d t}{\hbar} \sum_{\alpha} d W_{\alpha \nu}(t) A_{\alpha} \psi_{\nu} \otimes \chi_{\nu} & +\sum_{\alpha} d W_{\alpha \nu}(t) B_{\alpha} \psi_{\nu} \otimes \chi_{\nu} \\
& -\frac{i d t}{\hbar} \sum_{\alpha} A_{\alpha} B_{\alpha} \psi_{\nu} \otimes \chi_{\nu} .
\end{aligned}
$$

In the last term of (3.39) we have once again used the relation of the random variables (3.35). And finally we substitute (3.39) into (3.37) to arrive at

$$
d R(t)=-\frac{i d t}{\hbar}[H, R(t)]+d S(t)
$$

Here we have defined $d S(t)$ as

$$
d S(t) \equiv d T_{1} R(t)+R(t) d T_{2}^{\dagger}
$$

with

$$
d T_{\nu}=\sum_{\alpha}\left(-i d t A_{\alpha}+B_{\alpha}\right) d W_{\alpha \nu} .
$$

According to (3.41) and (3.42) the average at any given time of the Gaussian increments gives $E(d S)=0$ which leaves us with the desired von Neumann equation when taking the average at 
both sides of equation (3.40). This is the definitive proof that the two stochastic Schrödinger equations (3.33) and (3.34) reproduce the exact dynamics of any given system interacting with an environment.

\subsubsection{Determination of the observables}

Another important aspect that should be discussed before moving forward is the calculation of observables with this method. Assuming that we performed $N$ realizations, then the total density matrix can be expressed as

$$
\rho(t)=\frac{1}{N} \sum_{r}\left|\psi_{1}^{r}(t)\right\rangle\left\langle\psi_{2}^{r}(t)|\otimes| \chi_{1}^{r}(t)\right\rangle\left\langle\chi_{2}^{r}(t)\right|
$$

where $r$ denotes a particular realization. Due to the separability of the system and the environment the reduced density matrix is easily obtained as

$$
\rho_{S}(t)=\frac{1}{N} \sum_{r}\left|\psi_{1}^{r}(t)\right\rangle\left\langle\psi_{2}^{r}(t)\right|\left\langle\chi_{2}^{r} \mid \chi_{1}^{r}\right\rangle .
$$

Of course, the reduced density matrix of the system contains all the relevant information of the behavior of the system, in this case determined by the coupling with the environment. For completeness, the expected value of any observable of the system is simply

$$
\langle\hat{O}(t)\rangle=\frac{1}{N} \sum_{r}\left\langle\psi_{2}^{r}(t)|\hat{O}(t)| \psi_{1}^{r}(t)\right\rangle\left\langle\chi_{2}^{r} \mid \chi_{1}^{r}\right\rangle .
$$

\subsubsection{Important features of the method}

There are several interesting aspects to notice in the previous discussion. First of all, the system and environment evolve independently leading to the reduction of the total Hilbert space dimension to $H=H_{S} \oplus H_{E}$. In some cases (i.e. A thermal reservoir) following all the degrees of freedom of the environment would lead to additional difficulties making it an impossible task to perform any calculation, in particular one could not calculate explicitly (3.34). Fortunately, as it was shown in section 3.2.3 one does not need to know the entire state vector of the environment at any given time, but only the internal product $\left\langle\chi_{2}^{r} \mid \chi_{1}^{r}\right\rangle$.

As we mentioned at the beginning of this chapter, there is no particular restriction on the type of coupling and the type of system and environment that could be studied. 
Contrary to other standard methods, the evolution of two different and independent set of state vectors for the ket and bra includes all the non-Markovian effects induced by the coupling with the environment $[7,10,11,24]$ even though each individual evolution is completely Markovian. The reader should not be mislead by the latter observation; the stochastic term in the evolution has no physical meaning, moreover, the evolution of the system and environment state vectors are fictitious. In fact, the stochastic Gaussian variable is nothing more than a mathematical tool in order to recover the Liouville equation.

Alternative stochastic schemes can be carried out, as the quantum jumps in the piecewise deterministic process (PDP) presented by Breuer [10], where the Gaussian random variables are replaced by Poissonian increments. These random numbers can take two possible values, zero or one, leaving the system unchanged for a period of time, until the Poisson increment becomes one and the operators act changing the general state of the system, resulting in what is called a quantum jump. More recently, Lacroix [11] proposed several procedures to optimize the stochastic trajectories that result in the diffusion process similar to the method presented here. We will use this results and show qualitative in a further example, how the inclusion of a mean-field evolution on top of the stochastic dynamics results in the reduction of fluctuations and more accurate predictions in a larger time scale.

In Figure (3.5) we illustrate the basic ideas of this method. The total Hamiltonian is (3.29). It should be inferred from this discussion that the system and environment in the individual evolution have no information about the other. In a very practical way, the system has no knowledge of the presence of the environment and viceversa, they only perceive the action of the individual operators $\hat{A}(t)$ and $\hat{B}(t)$. As it is shown in (3.5) for an initial separable total state vector, the individual vectors follow independent stochastic quantum trajectories. These trajectories, when averaged in the ansatz of the density matrix, reproduce the total state at any time.

In conclusion, in this stochastic scheme the central quantum system, however decoupled from its sourrounding in every individual trajectory, will feel the effect of the enviornment through a driven noise term, allowing in this way to obtain the exact reduced density matrix at any given time.

\subsubsection{The Jaynes-Cummings model}

As an instructive exercise, we solve the well known Jaynes-Cummings model using the stochastic approach above presented. However no extra physical information will be obtained, some general features of the method can be shown explicitly, and the reader should find it straightforward to extend these procedures to systems presented in oncoming sections.

In the Jaynes-Cummings model we have the interaction of a single two-level atom with a single-mode field. The Hamiltonian in the interaction picture [30] can be written as 


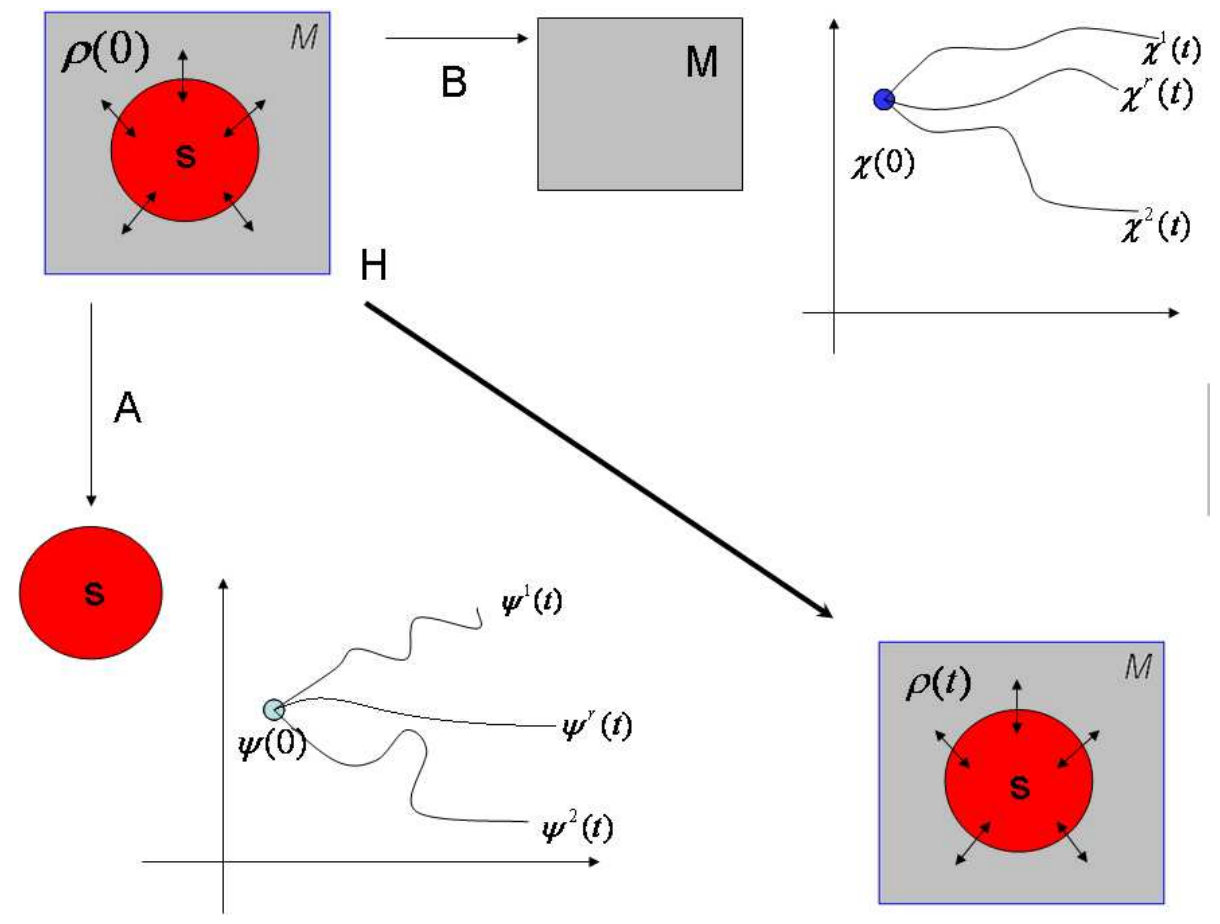

Figure 3.5: Schematic description of the separate evolution. At time zero, system and environment are separable, each one evolves independently under the action of their respective operators. The stochastic trajectories when averaged reproduce the total density matrix at any time. 


$$
H_{I}(t)=g\left(\sigma_{+} a e^{i \Delta t}+\sigma_{-} a^{\dagger} e^{-i \Delta t}\right)
$$

where $\sigma_{+}$and $\sigma_{-}$are the rising and lowering operators acting on the atom, $a$ is the operator of the field and the strength of the interaction is $g$.

In this example, the system is represented by the atom and the environment by the field. From (3.46), let us choose the operators of the system as

$$
\begin{aligned}
& A_{1}=\sigma_{+} \\
& A_{2}=\sigma_{-}
\end{aligned}
$$

and the environment

$$
\begin{array}{r}
B_{1}=g a e^{i \Delta t} \\
B_{2}=g a^{\dagger} e^{-i \Delta t} .
\end{array}
$$

In the stochastic evolution there is no need to write the total state vector since all the information is contained in the individual evolution of the system and environment. The state of the atom at any time is

$$
\left|\psi_{\nu}(t)\right\rangle=c_{a \nu}(t)|a\rangle+c_{b \nu}(t)|b\rangle
$$

and the state of the field in the Fock state basis can be expressed as

$$
\left|\chi_{\nu}(t)\right\rangle=\sum_{n=0}^{\infty} c_{n \nu}(t)|n\rangle .
$$

There are some aspects that we would like to comment before moving on. The Hamiltonian (3.46) clearly conserves the total number of excitations. This means that if the field at time zero has a well defined number of photons $M$ and the atom is in the excited state, the field at any given time will have at most $M+1$ photons. This kind of interpretation cannot be inferred directly from the state vector of the field. 
Let us make this idea clear. The individual evolution of the state vectors is given by (3.33-3.34), introducing (3.49) and (3.50) we obtain the Stochastic Diferential Equations (SDE) for the amplitudes $c_{a}, c_{b}$ and $c_{n}{ }^{4}$

$$
\begin{gathered}
c_{a \nu}(t+d t)=c_{a \nu}(t)-i c_{b \nu}(t) d W_{2 \nu}(t) \\
c_{b \nu}(t+d t)=c_{b \nu}(t)-i c_{a \nu}(t) d W_{1 \nu}(t) \\
c_{n \nu}(t+d t)=c_{n \nu}(t)+g\left(\sqrt{n} c_{n-1, \nu}(t) d W_{1 \nu}(t) e^{-i \Delta t}+\sqrt{n+1} c_{n+1, \nu}(t) d W_{2 \nu}(t) e^{i \Delta t}\right) .
\end{gathered}
$$

The idea is to give unquestionable evidence that this evolution individually has no physical interpretation. For the case of the number of photons in the field, after a finite time $c_{n}$ takes non-zero values for every Fock state, and it is not restricted to $M$ and $M+1$. Moreover, if one turns off the interaction between the field and the atom $(g=0)$ one would expect $c_{a}$ and $c_{b}$ to maintain their initial value, and as it is shown in (3.51) they do not. This is depicted in Figure 3.6, where the atom is initially in the excited state. As shown, the probability of finding the atom in the ground state is always zero, while the individual average of $\left\langle c_{b 2}^{*} c_{b 1} / c_{2}^{*} c_{1}\right\rangle$ have finite values. Basically, we need to reinstate an important feature of this method that was mentioned earlier: in average, the total density matrix will be reproduced but the individual evolution of the central quantum system is not a real physical process.

For simplicity, we will assume that the detuning is zero $(\Delta=0)$. We want to calculate the probability of finding the atom in the ground state $P_{b}(t)=\left\langle b\left|\rho_{S}(t)\right| b\right\rangle$, for the field initially empty and the atom in the excited state. This expression can be analytically obtained as simply $P_{b}(t)=$ $\sin ^{2} g t$. In (3.7), results for $P_{b}(t)$ are presented. With a reasonable small number of simulations $\left(10^{4}\right)$ and a very short computational time the exact results are recovered by the stochastic scheme. It is also important to notice that with the help of mean-field, convergence is obtained for longer times. Even though is not in the scope of this work (details will not be presented here) the numerical integration of the SDE (3.51-3.52) and during the rest of this document, it were performed using a special Runge-Kutta (RK) method for stochastic equations [31 $]^{5}$.

\footnotetext{
${ }^{4}$ The separability that was imposed once again appears in these equations, since the amplitudes for the atom and field evolve independently.

${ }^{5}$ In this paper the author presents an efficient numerical method for solving SDEs. He shows that a usual RK 4 scheme for deterministic differential equations is transformed into a RK 2 in order to solve SDEs.
} 


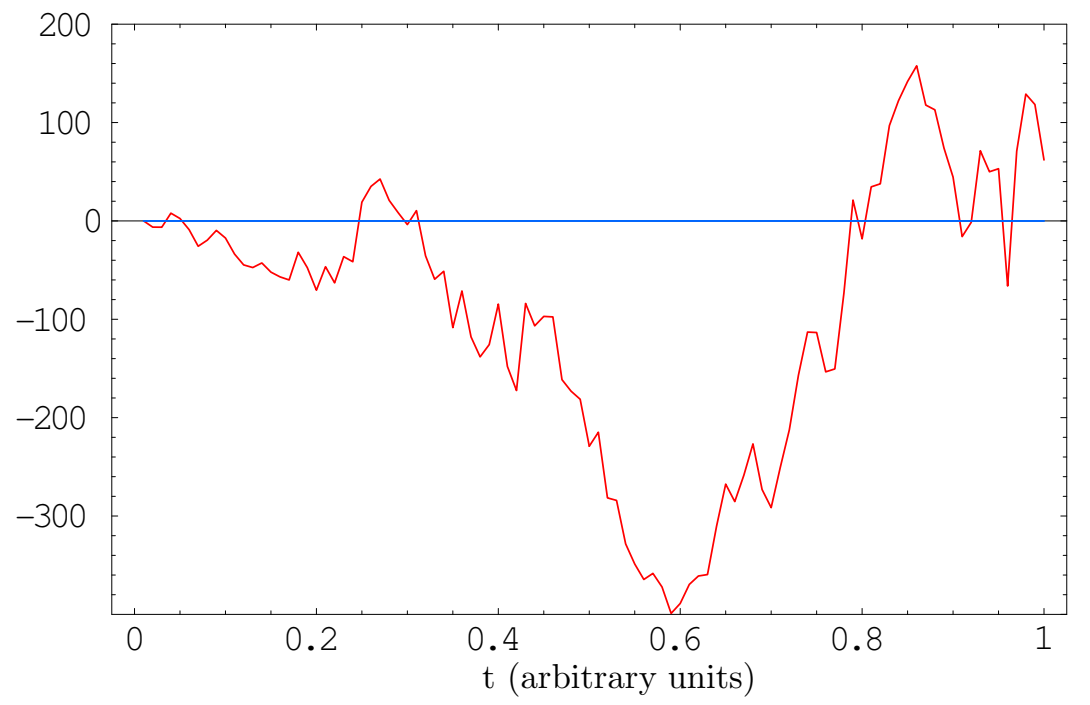

Figure 3.6: Comparisson between the individual average of $\left\langle c_{b 2}^{*} c_{b 1} / c_{2}^{*} c_{1}\right\rangle$ (red), and the ground state probability average from the reduced density matrix $P_{b}(t)=\left\langle b\left|\rho_{S}(t)\right| b\right\rangle$ (blue). No field coupling $g=0$ and the atom initially in the excited state. Results shown for $10^{4}$ realizations.

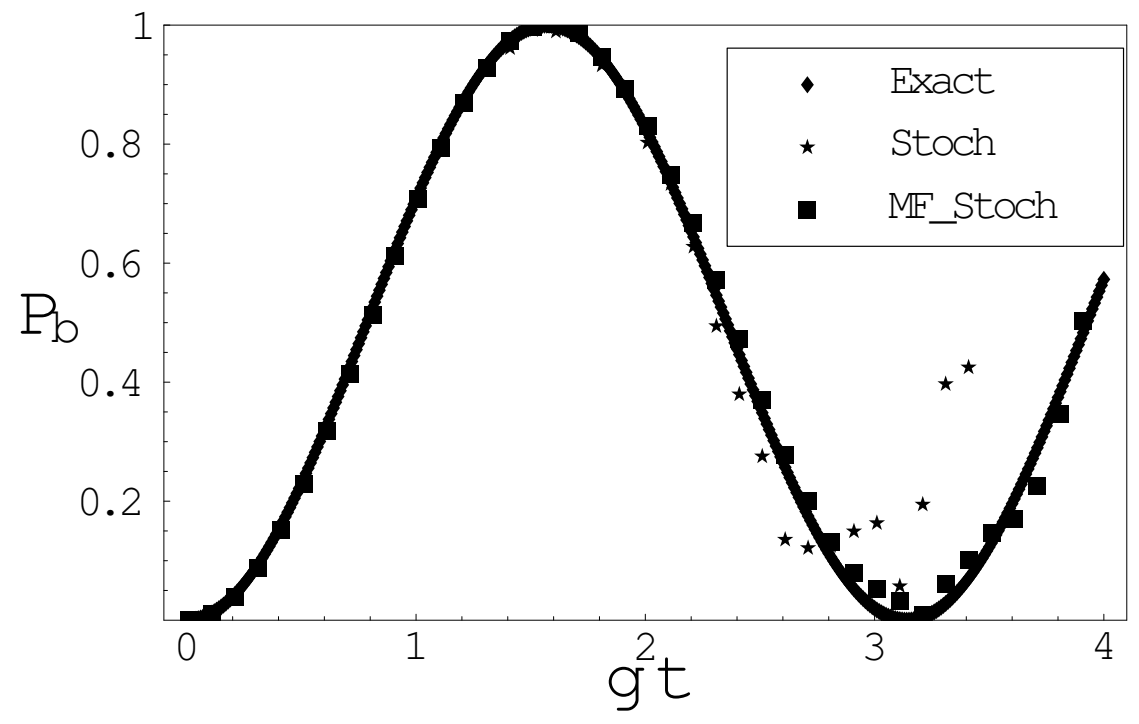

Figure 3.7: Probability of finding the atom in the ground state vs.time. The solid line represents the exact solution, the stars give the stochastic results and the boxes present the stochastic results including Mean-Field. This calculation was performed with $10^{4}$ realizations. 


\subsubsection{Two level system (qubit) coupled with a thermal bath}

Another important model that has been extensively studied in the literature is a two level system interacting with an infinite number of external modes at thermal equilibrium. The role of this example is not only to illustrate the advantages of the method but to engage in a physical problem that is relevant in the case of microcavity polaritons, that is, how to model the effects of a thermal bath in a given system.

The hamiltonian for this system is as follows

$$
H=\sigma_{+} B(t)+\sigma_{-} B^{\dagger}(t)
$$

where the operators acting on the bath are $B(t)=\sum_{k} g_{k} b_{k} e^{i\left(\omega_{0}-\omega_{k}\right) t}$. Another important aspect that arises in this example is the fact that the operators on the system are exactly the same as in the Jaynes-Cummings model. In other words, the evolution of the state vector for the two level atom follow 3.51. This is a general consequence of the stochastic approach. Since the system and environment evolve independently, if the operators of the system remain unchanged and we consider a different environment, the new dynamics will be obtained only by calculating the new SDE for the state vector of the environment. The system only perceives the type of environment and coupling when the average is performed. This suggests that an alternative modular calculation is also possible, but no further discussion on this will be presented here.

Similar to the previous example we are now interested in calculating the time evolution of the excited state population of the atom. Above, we calculated explicitly the state vector of the field at all times (3.52), but as it was mentioned in an earlier discussion, in order to calculate an observable for the system we only need to know the inner product of the environment vectors (3.45). This may be interpreted as equivalent of not the entire evolution of the environment state would be relevant in the effect on the system.

For an atom initially in the excited state and all the modes empty (or the thermal bath at zero temperature) the total state vector is $|\Phi(0)\rangle=|a\rangle \otimes|0\rangle$. The evolution of the environment state vector is

$$
\begin{array}{r}
\left|\chi_{\nu}(0)\right\rangle=|0\rangle \\
\left|\chi_{\nu}(d t)\right\rangle=|0\rangle+B^{\dagger}(0)|0\rangle d W_{1 \nu}(0) \\
\left|\chi_{\nu}(2 d t)\right\rangle=|0\rangle+\ldots+B(d t) B^{\dagger}(0)|0\rangle d W_{1 \nu}(0) d W_{2 \nu}(d t),
\end{array}
$$

the inner product at time $t$ for a particular realization will always be proportional to 


$$
\left\langle\chi_{2}(t) \mid \chi_{1}(t)\right\rangle \propto\left\langle 0\left|B(t) B^{\dagger}(t)\right| 0\right\rangle
$$

The right hand side of equation (3.55) is the Bath Correlation Function (BCF). As it is shown in (3.54), the stochastic terms will still be present in order to couple with the evolution of the atom to construct the total density matrix. In this particular example the $\mathrm{BCF}$ is known analytically

$$
\left\langle 0\left|B(t) B^{\dagger}(t)\right| 0\right\rangle=\int d \omega J(\omega) \exp \left(i\left(\omega_{0}-\omega\right)(t-t)\right)
$$

in terms of the bath spectral density $J(\omega)$. For the damped on resonance Jaynes-Cummings model this quantity is given by

$$
J(\omega)=\frac{1}{2 \pi} \frac{\gamma_{0} \lambda^{2}}{\left(\omega_{0}-\omega\right)^{2}+\lambda^{2}}
$$

Here we can interpret $\gamma_{0}^{-1}$ as the Markovian relaxation time of the open system and $\lambda^{-1}$ as the correlation time of the reservoir. This quantities contain all the information on the effect of the reservoir on the atom. The practical value is that there is no need to calculate the total state vector of the reservoir, task that would have been as difficult as to numerically integrate the total problem at the first place.

In figure (3.8), the excited state probability is presented $P_{a}(t)=\left\langle a\left|\rho_{S}(t)\right| a\right\rangle$. For a reasonable time, the exact evolution is recovered. In particular, the first collapse and revival, which is a purely quantum effect, is reproduced. This clearly shows that the scope of the present method is beyond Weisskopf-Wigner or any Markovian approximation, because the latter ones can only reproduce an exponential decay.

\subsection{Exact reformulation of interacting bosons in the pres- ence of an environment}

Up to this point we have presented two stochastic methods developed recently in the literature. The first one is able to recover the exact evolution of a closed system when particle interactions are considered. The second one takes into account the coupling of a system with its surroundings. Even though thermal equilibrium can be studied as shown in section (3.1.4) one resigns the idea of reviewing dynamical behavior. Because of the short lifetime of the polaritons, a dynamical picture is necessary and the last possibility has to be neglected. 

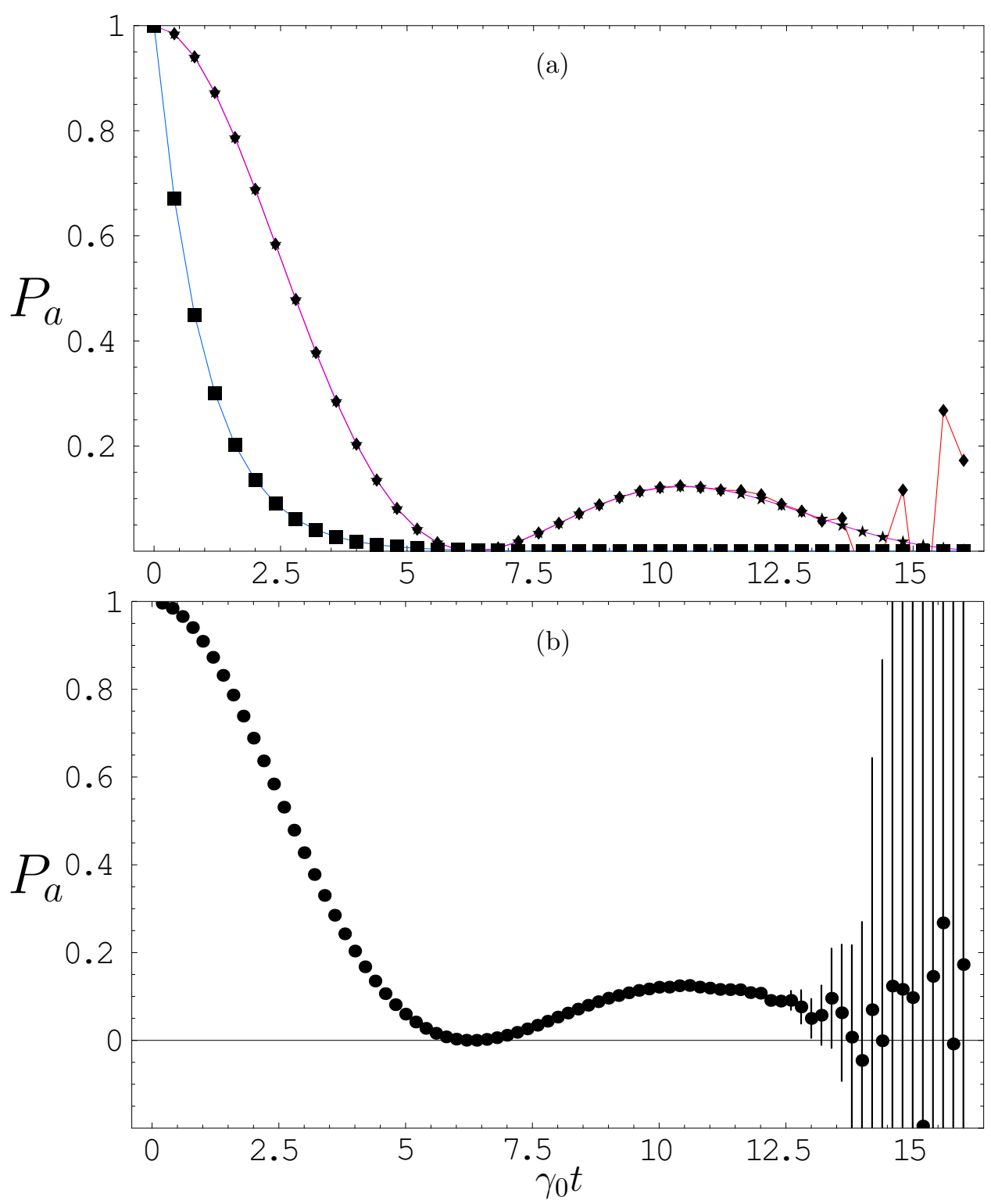

Figure 3.8: Evolution of the excited state probability. The results were obtained with $10^{8}$ simulations. (a) Comparisson between the analytical solution (stars), the stochastic diffusive evolution (diamonds) and the solution with the Born-Markov approximation (squares) [10] for $\gamma_{0} / \lambda=5$. As it will be usual for stochastic schemes the convergence of the solution will be time limited. (b) Stochastic result with its standard deviation. 
In this section we present a new stochastic approach. It uses the two main ideas of the methods discussed above, and it is able to recover the exact dynamics in a system of interacting bosons coupled with an environment, leading us a step closer to study the dynamical behavior of microcavity polaritons.

In the one hand, Breuer proposed a method which enables us to separate the evolution of the system and environment state vectors. On the other, it has been proven that the evolution of a system composed by $\mathrm{N}$ interacting bosons, can be changed to a stochastic differential equation for a vector in the single particle Hilbert space.

We will show that if the system vector is written as the Hartree-Fock ansatz (the $\mathrm{N}$ bosons in the same single particle state), the problem of $\mathrm{N}$ interacting bosons coupled with an environment transforms into the evolution of one particle in the presence of an environment, where the set of stochastic equations differential equations is decoupled. In other words, we not only separate the evolution of the system and environment but reduce the $\mathrm{N}$ interacting particles to just one particle dynamically evolving in a stochastic manner.

\subsubsection{Stochastic solution}

The total Hamiltonian of the problem we want to study has the form

$$
H_{T}=H_{S}+H_{E}+H_{i}
$$

where $H_{S}$ is the Hamiltonian of the system ${ }^{6}$ (3.1), $H_{E}$ the Hamiltonian of the environment and $H_{i}=\sum_{\alpha} A_{\alpha} B_{\alpha}$ the coupling between system and environment. In the Schrödinger picture the evolution of the state vectors (3.33) and (3.34) are easily calculated and can be written as

$$
d \psi_{\nu}(t)=-i d t H_{S} \psi_{\nu}-i d t \sum_{\alpha} d W_{\alpha \nu}(t) A_{\alpha} \psi_{\nu}(t)
$$

and

$$
d \chi_{\nu}(t)=-i d t H_{E} \chi_{\nu}+\sum_{\alpha} d W_{\alpha \nu}(t) B_{\alpha} \chi_{\nu}(t)
$$

These equations when averaged together in the density matrix, reproduce the Liouville equation.

\footnotetext{
${ }^{6}$ It contains the particle-particle interactions, free energy and possible confinement. See equation (2.12)
} 
This is demonstrated in the same manner as for the interaction picture. Recalling from the coupling between polaritons and phonons (2.16) the operators $A_{\alpha}$ take the form

$$
A=p_{k_{1}}^{\dagger} p_{k_{2}}=\iint d x d x^{\prime} u_{k_{1}}(x) u_{k_{2}}^{*}\left(x^{\prime}\right) \Psi^{\dagger}(x) \Psi\left(x^{\prime}\right)
$$

If we now assume that the system is composed by $\mathrm{N}$ bosons, its state vector can be written as $\psi=|N: \phi\rangle$. In a similar way as for the closed system, we need to find an equation of motion for $\phi$, and prove that combined with the evolution of the environment state vector can in fact reproduce the Liouville equation.

Firstly, equation (3.59) becomes

$$
d\left|N: \phi_{\nu}\right\rangle=-i d t H_{S}\left|N: \phi_{\nu}\right\rangle-i d t \sum_{\alpha} d W_{\alpha \nu}(t) A_{\alpha}\left|N: \phi_{\nu}\right\rangle
$$

where we have already calculated explicitly $d\left|N: \phi_{\nu}\right\rangle$ in (3.10), and the first term in the right hand side in (3.6). With the explicit form of the operators associated with the coupling with the environment (3.61), we calculate the last term and obtain

$$
A_{\alpha}\left|N: \phi_{\nu}\right\rangle=\sqrt{N}\left[\int d x \tilde{a}_{\alpha} \phi(x) \Psi^{\dagger}(x)\right]|N-1: \phi\rangle .
$$

In this notation, $\tilde{a}_{\alpha}$ is the operator associated with $A_{\alpha}$ acting directly into the single particle state. The summation over alpha is introduced in (3.63). We now need to compare both sides of equation (3.59): The infinitesimal change in the Hartree-Fock state vector (3.10) with the action from both the system Hamiltonian (3.6) and the operators $A$ associated with the environment coupling (3.63).

This is in principle the same task we performed for the closed system stochastic approach in section (3.1), including a new element. The last one is related to the noise term $d W_{\alpha \nu}$ that is introduced when the system and environment state vectors are split. Then, the evolution of the single particle state is

$$
d \phi_{\nu}=-i d t G_{\nu}-i \sum_{\alpha} \tilde{a}_{\alpha} \phi_{\nu} d W_{\alpha \nu}(t)+d M_{\nu}
$$

In (3.64), $G$ corresponds to the Mean-Field evolution (3.7) associated with the particle interactions, $d B(x)$ is the stochastic noise term responsible for reproducing the quadratic terms in (3.6) that are 
left out from making $d \phi$ proportional to $d t$. It includes information from the interactions that are not contained in $G$. And finally, the second term in (3.64) carries the net effect of the presence of an environment and its respective coupling with the system.

The stochastic differential equation for the single particle state describing the system's evolution (3.64), together with the evolution for the environment state vector, contains all the information from the problem of interest. In order to prove that this statement is correct, it is necessary to verify that, when averaged in the anstaz for the density matrix they reproduce the Liouville equation. This mathematical procedure is identical to the one already presented in 3.2.2.

In conclusion, we have reduced the problem of $\mathrm{N}$ interacting bosons coupled with an environment, to the independent evolution of a single particle and the environment. The gain obtained by this incredible simplification is to be paid by the fact that two separate stochastic processes were introduced and a large number of realizations have to be performed in order to converge to the exact solution. The reach of the method is tested with an example in the next section, and we will prove it's usefulness to engage in the microcavity polariton problem.

\subsubsection{N-bosons in a two level system coupled with an external mode}

The model is an extension of the system introduced in section 3.1.3. We will consider $\mathrm{N}$ bosons in two possible modes with contact interactions in the $\mathbf{k}$ space. The two modes are coupled through an intermediary oscillator (picturing a phonon). The Hamiltonian is

$$
H=\sum_{k=0,1} E_{k} a_{k}^{\dagger} a_{k}+E^{\prime} b^{\dagger} b+\Omega\left(b^{\dagger} a_{0}^{\dagger} a_{1}+a_{1}^{\dagger} a_{0} b\right)+\kappa\left(a_{1}^{\dagger 2} a_{1}^{2}+a_{0}^{\dagger 2} a_{0}^{2}\right)
$$

where $b$ is the annihilation operator for the phonon. The constants $\Omega$ and $\kappa$ are the coupling and interaction strength respectively. Mode $k=0$ will be associated with the ground state, and $k=1$ with the excited state. The two nonlinearities in (3.65) do not allow for an analytical solution. We apply the stochastic methodology and compare its predictions with the results obtained by direct numerical integration.

In order to gain a qualitative idea of the convergence of the method, we find the evolution of the system for different parameters, in particular we change the number of particles and the interaction strength. In this simulations we have considered zero detuning $\Delta=\left(E_{1}-E_{0}\right)-E^{\prime}=0$ between the two level and the external field. In figure (3.9) the occupation probability of the ground state is calculated $P_{0}=\left\langle p_{0}^{\dagger} p_{0}\right\rangle$, for an initial state where all the particles are in the excited state and the external field is empty. The interaction strength is chosen to be $\kappa=0.01 \Omega$ and we vary the total number of particles. In the case $N=1$, the system is analog to the Jaynes-Cummings model 

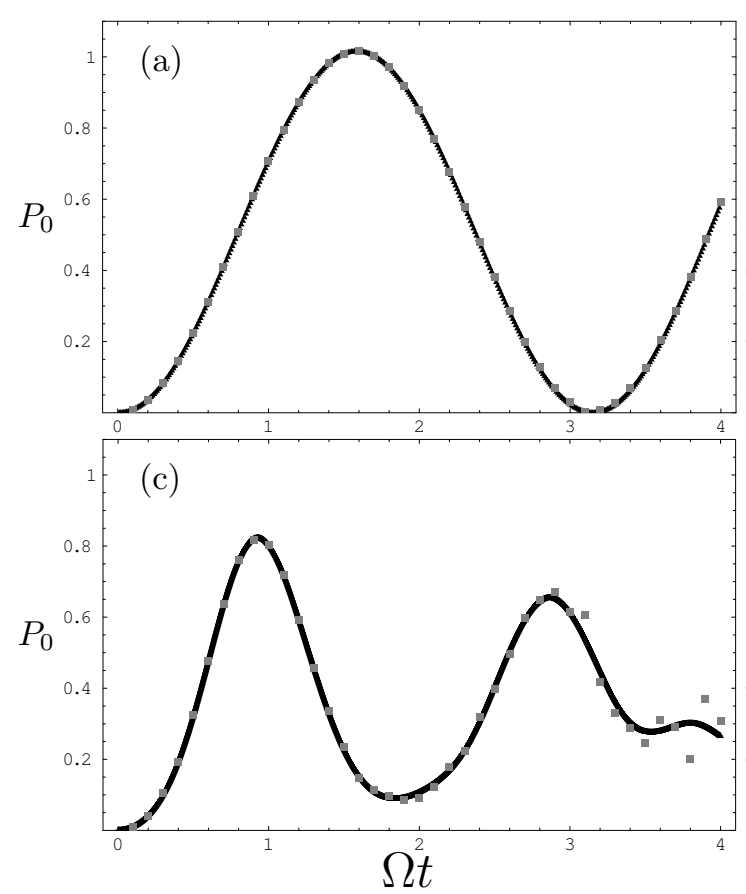

(b)

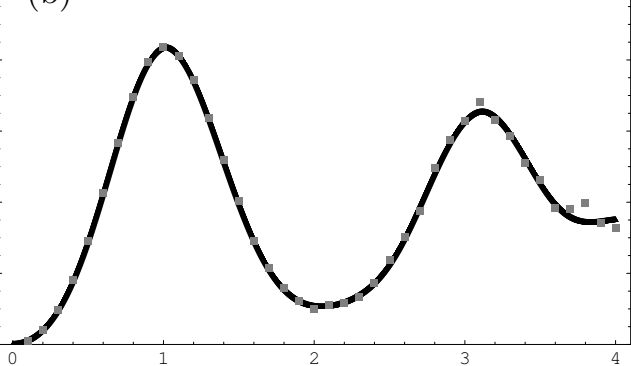

(d)

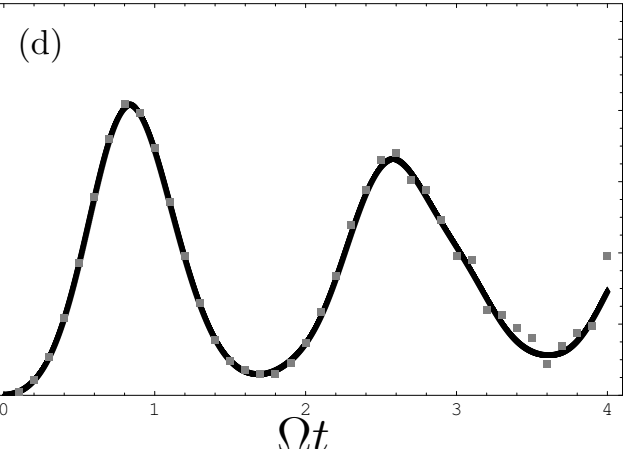

Figure 3.9: Occupation probability of the ground state $P_{0}=\left\langle p_{0}^{\dagger} p_{0}\right\rangle$ for different number of particles $\mathrm{N}=1$ (a), 5 (b), 7 (c) and 10 (d). The system is initially in the excited state and the field empty. Comparisson between the results obtain through direct numerical integration (solid line) and the stochastic predictions (squares). Results obtain with $5 \times 10^{5}$ simulation for an interaction strength of $\kappa=0.01 \Omega$.

and the same results are recovered. Similar results are presented in Figure (3.10), for a maximum number of particles $N=10$ (where we show the standard errors) with the interaction strength increased to $\kappa=0.1$. As the number of particles increases the method becomes computationally demanding in terms of the required number of realizations for reaching a good convergence.

While the chosen interactions strengths, between particles and with the external field, are not describing any physical process in particular, the method proved to be a very practical tool in the dynamical study in highly non-linear systems. In systems such as the microcavity polariton, for a small wave vector, the particle-particle interaction can be approximated by a contact potencial (see Chapter 2.2). This is several orders of magnitude smaller than the non-physical interaction strength $\kappa$ presented in this section. After taking into account this fact, a larger number of polaritons might be considered. In the next Chapter we will use the present stochastic scheme to study the dynamics of microcavity polaritons. 


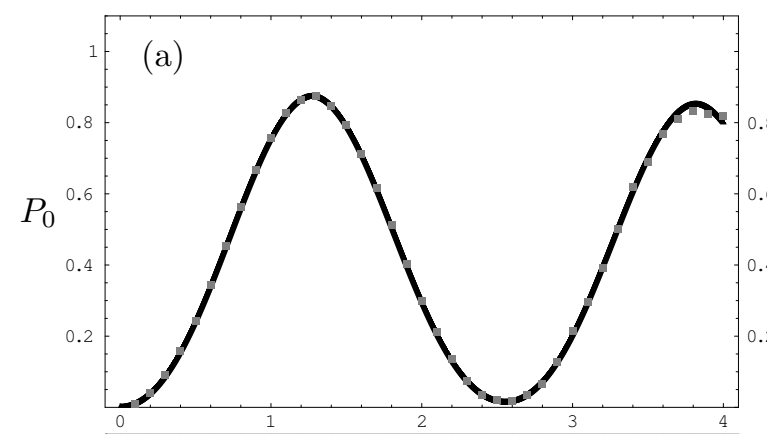

(b)
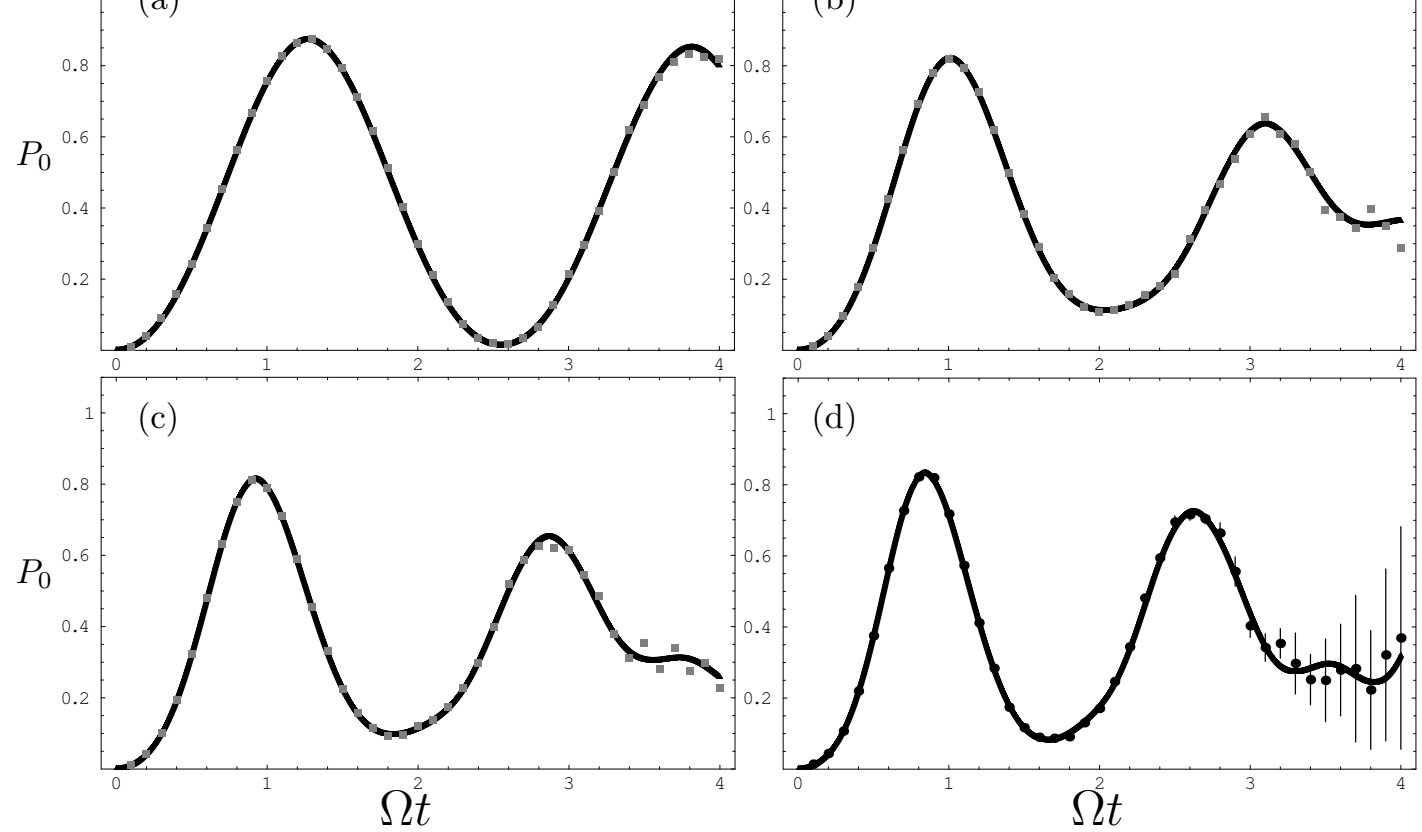

Figure 3.10: Occupation probability of the ground state $P_{0}=\left\langle p_{0}^{\dagger} p_{0}\right\rangle$ for different number of particles $\mathrm{N}=2$ (a), 5 (b), 7 (c) and 10 (d). The system is initially in the excited state and the field empty. Comparisson between the results obtain through direct numerical integration (solid line) and the stochastic predictions (squares). Results obtain with $10^{6}$ simulation for an interaction strength of $\kappa=0.01 \Omega$. In plot (d) the standard deviation is shown. 


\section{Chapter 4}

\section{Spontaneous coherence build-up and dynamics of Bose-Einstein phase transition in microcavity polaritons}

As we stated in the introduction, our main goal was to study the dynamical behavior of microcavity polartions. So far we have presented a new stochastic scheme that permits the study of the full quantum effects involving a bosonic interacting system coupled with its environmnet. With this powerfull tool, we will engage in the more complex problem of polariton dynamics.

The acumulation of polaritons in the ground state or polariton condensation has been the recent focus of several studies, both theoretical [4, 17] and experimental [5]. Theoretically, most authos favored a semiclassical Boltzmann equation [17, 18, 20], where the polariton relaxation is described $^{1}$. Also, the influence of polariton interactions on the polariton-laser linewidth has been equally adressed [6]. The Born-Markov approximation is performed in this papers, but its validity extends only to the low density regime and important correlations are neglected.

In section 4.1 we developed a few level model of polariton dynamics which includes the polaritonpolariton Coulomb interaction and the polartion-phonon scattering on equal grounds. In order to fully study the dynamics and statistics of the acumulation of polaritons in the groound state, we not only calculate the population buildup but also the second order coherence degree $g^{(2)}(0)$. The last, gives clear information for both polariton statistics and properties of the emmited light

\footnotetext{
${ }^{1}$ In the time range of polariton lifetime
} 
after recombination. We will show that Coulomb interaction is highly responsable in the rising of coherence while phonon scattering mediates the relaxation dynamics. In this sense, a posible phase transition is triggered by quantum fluctuations via polariton-polariton interactions.

Another important aspect that will be studied in 4.2, is the spontaneous parametric scattering of microcavity polaritons. In this process pump polaritons are scatter in pairs (signal and idler) according to momentum and energy conservation criteria. The resulting emission is ring shaped and has been recently observed [2]. This process has open a way to efficiently create correlated polaritons, which in consecuence produce correlated photons in their radiative decay, relevant for quantum computation applications. Even though qualitative agreement was found between previously existing theory $[12,13]$ and the experimental results, additional observed properties such as the dephasing of the polaritons, specially the idler states, are not accounted in the theory where high order correlation terms are not considered. We show that our stochastic approach reproduces the observed experimental results. Also, when the pumped population is increased above some threshold level, an exponential rise in signal and idler population is observed, mediated by the Coulomb scattering.

The advantages of the new stochastic approach from previous work is manyfold: First of all, we keep track of the complete fluctuations from the polariton field. Even though we start with a few level mode, we will see that such approximation is not needed, and the parametric emission into all available modes is automatically taken into account, as well as correlations between all modes.

\subsection{Dynamical condensation of microcavity polaritons}

In a typical photoluminescence experiment under nonresonant excitation, the strong energy dispersion of microcavity polaritons leads to a bottleneck relaxation dynamics $[18,20]$, where polaritons go to the flat exciton-like region of the energy dispersion curve. From this point on, polaritons relax into the bottom states of the lower branch. Using these considerations a simplified model of polariton dynamics is an effective two-level system [3], where the ground state corresponds to $\mathbf{k}=0$ which is non-degenerate $\left(E_{0}\right)$, and the excited or bottleneck set of states is macroscopically degenerate $\left(E_{1}\right)$ (see Figure 4.1).

In the lower polariton branch the Hamiltonian describing both Coulomb and phonon scattering is 2.18. Here the semiconductor environment is modelled by acoustical phonons distributed according to the lattice temperature. To recall the discussion from Chapter 2, the excitonic component of the polaritons is responsible for both Coulomb interactions and polariton-phonon decoherent scattering. The relevant material parameters were also introduced in this Chapter. The dynamical condensation of microcavity polaritons will be studied using the stochastic formalism presented previously. The quantization area $A=100 \mu m^{2}$ determines the Coulomb interaction (2.13) and the phonon coupling 


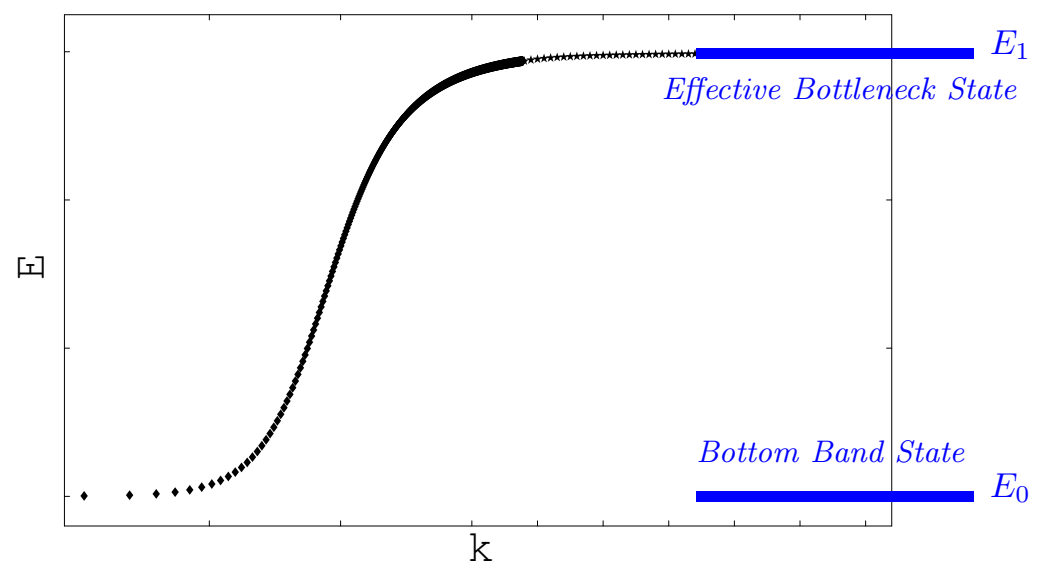

Figure 4.1: Lower polariton energy branch versus total momentum in a linear-log scale. The excited state is represented by the effective bottleneck state, which is at the edge of the flat exciton-like dispersion region. The ground state is the zero momentum state in the bottom of the dispersion curve.

terms (2.17).

We consider $\mathrm{N}$ interacting polaritons in the presence of a semiconductor environment at zero temperature. Since, the effective two level model does not consider the relaxation from high energy states into the effective bottleneck state, the initial state consists of the total number of polaritons randomly distributed in the excited energy level (importantly, no seed is needed for level $E_{0}$ ). Indeed, a zero temperature environment will force the polaritons into their ground state following a non-trivial evolution due to polariton interactions. Our main interest is to give a full description of the ground state statistics.

We first calculate the ground state probability distribution $P_{0}(n, t)$. This is obtained from the one-particle density matrix $\rho^{(1)}\left(x, x^{\prime}\right)=\left\langle\hat{\Psi}^{\dagger}\left(x^{\prime}\right) \hat{\Psi}(x)\right\rangle$

$$
P_{0}(n, t)=\frac{N !}{n !(N-n) !} E\left[\left(\phi_{02}^{*} \phi_{01}\right)^{n}\left\langle\phi_{12}^{*} \mid \phi_{11}\right\rangle^{N-n}\left\langle\chi_{2} \mid \chi_{1}\right\rangle\right]
$$

Where the state vector of the two level polariton system is simply $\left|N: \phi_{\nu}\right\rangle$, with the single particle vector $\phi_{\nu}(t)=\phi_{0 \nu}(t) u_{0}+\phi_{1 \nu}(t) u_{1}$. The environment is described by the state vector $\chi_{\nu}$ and recalling form the discussion of Section 3.2.4 its evolution is exactly the same as in the example of Section 3.2.6. Equation (4.3) should be interpreted as the probability of having exactly $N_{0}$ polaritons in the state $E_{0}$. This is initially a delta function centered at $n=0$, since all polaritons are initially in the excited state. For comparison purposes, an overall factor, $\gamma$, is introduced in the Coulomb interaction term in order to account for the effective strength of two body Coulomb interactions: $\gamma=0$ corresponds to no Coulomb interaction. As is clearly shown in Figure 4.2, for 

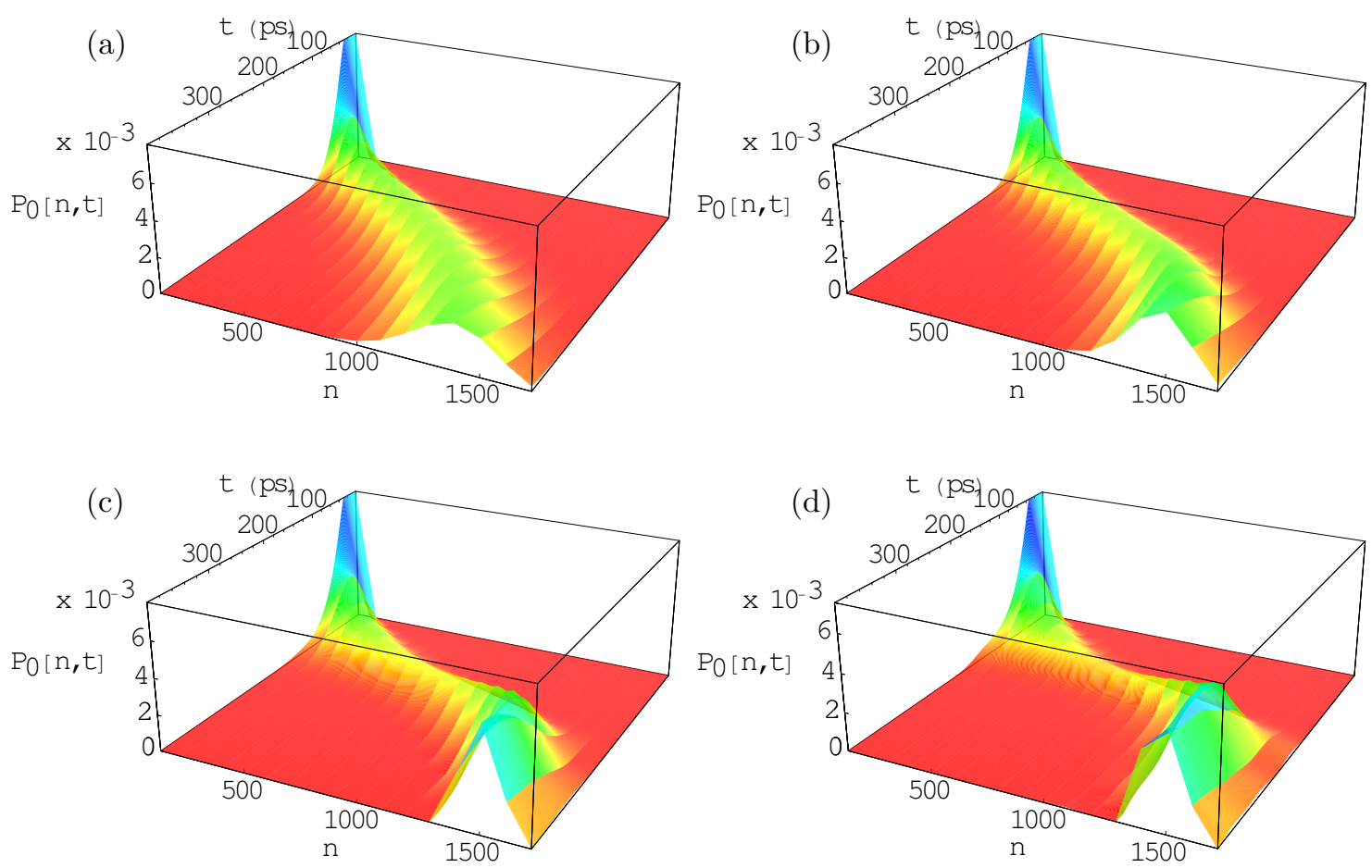

Figure 4.2: Ground state probability distribution $P_{0}(n, t)$ for different polariton interaction strengths: (a) $\gamma=0$, (b) $\gamma=0.4$, (c) $\gamma=0.8$ and (d) $\gamma=1$, averaged over $5 \times 10^{5}$ realizations. The total number of polaritons is $N=1600$ and lattice temperature $\mathrm{T}=0$.

a fixed total number of polaritons $N=1600$, the polariton system decays monotonically into its ground state when interactions are not included. As the interactions are turned on, the system's dynamics changes completely. Polaritons start with a slow relaxation dynamics, but after a finite time the ground state becomes rapidly populated with a macroscopic fraction of $N$.

The Gaussian-like distribution for vanishing inter-particle interactions is evidently different from the Poisson-like distribution when the interactions are fully included, as displayed in Fig.4.3. This latter feature is a clear signature of an interaction driven coherence buildup process for which an off-diagonal-long-range order (ODLRO) can develop. It is well known [15] that ODLRO does not implies a finite value of $\left\langle p_{0}\right\rangle$, in agreement with our results.

The so-called coherence degree parameter is defined as $\eta=2-g^{(2)}(0)$, with $g^{(2)}(0)=\left\langle p_{0}^{\dagger 2} p_{0}^{2}\right\rangle /\left\langle p_{0}^{\dagger} p_{0}\right\rangle^{2}$. This parameter takes values between 0 and 1 for states ranging from chaotic/thermal to coherent. Using this parameter the full statistical properties of the ground state are unravelled. Starting with an incoherent state ( $\mathrm{N}$ polaritons randomly distributed in the excited level), the coherence rises as the ground state becomes populated. This is clearly shown in Fig.4.4a. Again, the most inter- 

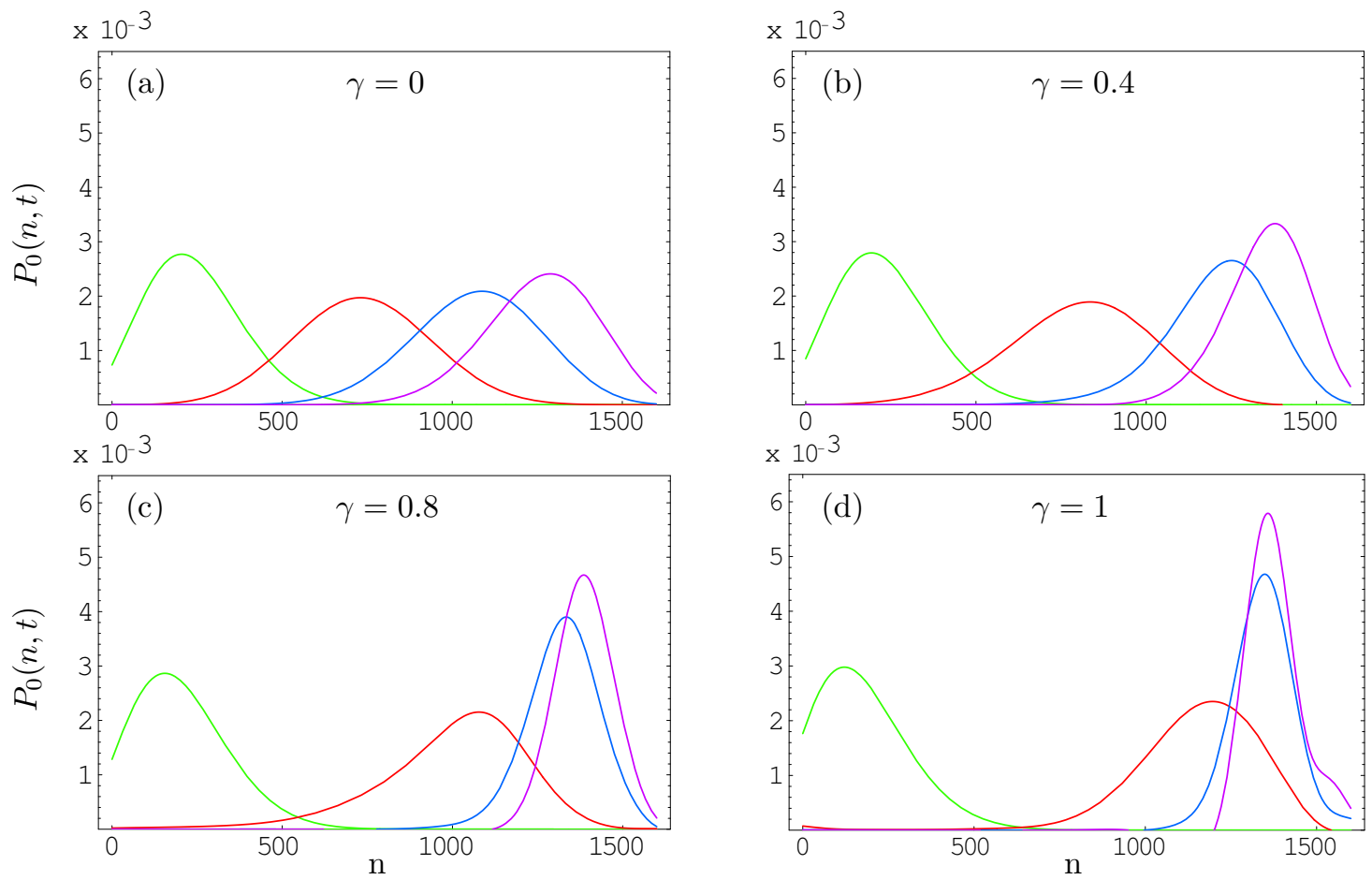

Figure 4.3: Projections of the ground state probability distribution $P_{0}(n, t)$ at four various times: $100 \mathrm{ps}$ (green), $200 \mathrm{ps}$ (red), $300 \mathrm{ps}$ (blue) and $400 \mathrm{ps}$ (purple). Results are shown for $N=1600$, $5 \times 10^{5}$ realizations and different polariton interaction strengths: (a) $\gamma=0$, (b) $\gamma=0.4$, (c) $\gamma=0.8$ and $(\mathrm{d}) \gamma=1$. 
esting observation about this evolution is the difference between the smooth growing of coherence in the non-interacting regime as compared with the sudden rise of coherence for the interacting case, complementing the results depicted in Fig.4.3. A threshold behavior is clearly observed in Fig.4.4b. Once the number of polaritons, or equivalently the density, is increased beyond a certain value the coherence rises suddenly. A possible phase transition triggered by Coulomb interactions is thus reinforced. Moreover, since no high order correlations are in principle discarded by the present method, as it is usually the case for condensed matter systems [23, 25] (excitons) and [6] (polaritons), we can quantify their influence on the polariton condensation dynamics.

Finally, we would like to remark some of the most important aspects of these results. First of all, the threshold behavior that governs the dynamics was already discussed in Ref.[4], and reported results are in good agreement with ours. This gives us confidence that the stochastic scheme we used is indeed a very practical tool for the study of complex systems like those found in semiconductor microcavities. In particular, effects due to the semiconductor environment are responsible for the relaxation of the system, leading to a continuous accumulation of polaritons in the same final state at low temperatures. Nevertheless, correlations generated by interactions play a crucial role in the polariton condensation dynamics. While a coherence degree parameter close to 1 can only be interpreted as a criteria for polariton lasing [4], instead of BEC in a quasi-two-dimensional nanostructure, signs of a true phase transition are present in our results as a consequence not only of the bosonic behavior but from quantum fluctuations provided by polariton interactions. Since the appearance of the phase transition is governed by Coulomb interactions, our results point toward a BEC type behavior rather than a polariton laser. 

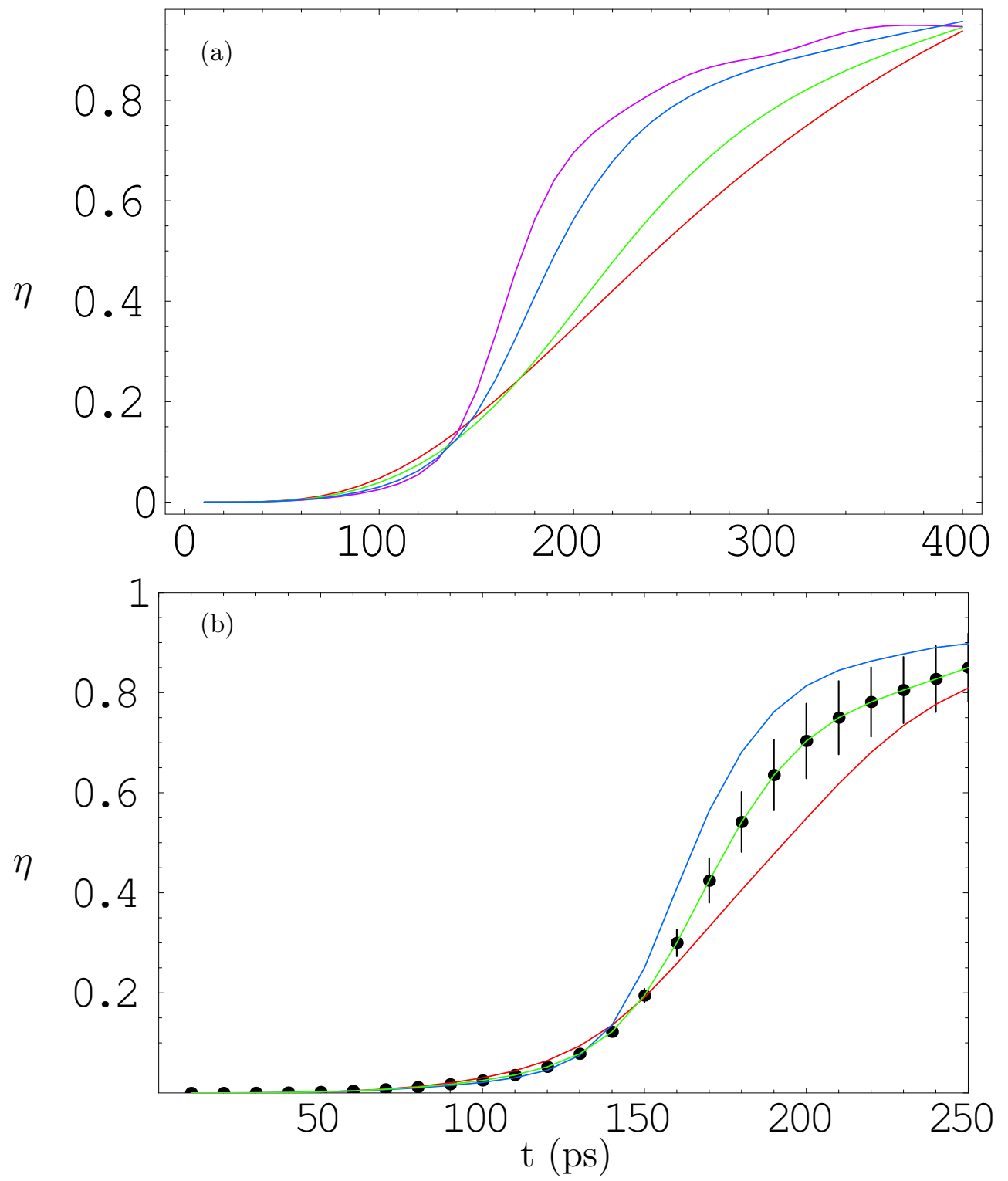

Figure 4.4: Time evolution of the coherence degree parameter $\eta$ : (a) $N=1600$ and different interaction strengths $\gamma=0$ (red), $\gamma=0.4$ (green), $\gamma=0.8$ (blue) and $\gamma=1$ (purple). (b) Different number of polaritons $(\gamma=1) N=1400$ (red), 1600 (green) and 1800 (blue). Lattice temperature $\mathrm{T}=0$ and $5 \times 10^{5}$ stochastic trajectories. Dispersion bars are shown for the intermediate case of $N=1600$. 


\subsection{Parametric down convertion}

In recent experiments $[2,3]$ polaritons are optically excited at a well defined in-plane momentum $\mathbf{k}$, allowing a direct control of their dynamics. The strong Coulomb interaction results in a spontaneous parametric scattering of polariton pairs (signal and idler) according to momentum and energy conservation criteria. For resonant optical excitation, the scattering leads into a final state 8shaped distribution in $\mathbf{k}$ space, as first suggested by Ciuti et al [12] and experimentally confirmed in Ref.[2]. We use the present stochastic scheme to study the dynamics of initially pumped polaritons in a particular momentum state $\mathbf{k}$ in the lower polariton branch. Once again, the advantages of the present approach are manifold. Similar to the condensation of polaritons studied in the previous Section, the theoretical discussion of parametric scattering has been based upon MFA, and thus the results are only valid below the threshold of parametric luminescence. Thus, the study of the macroscopic behavior of interacting polaritons in a relaxing environment has been poorly investigated [9]. Moreover, the dynamical behavior of condensed matter quasi-particles in an out of equilibrium regime can be systematically studied within our approach. In this Section we abandon the effective two-level approximation and resort to an extended multi-level description.

For the numerical simulations that follows, the total Hamiltonian is still given by Eq.2.18, where the system's parameters are the same as in Section 4.1. The possible final states for the scattering of two polaritons pumped at $\mathbf{k}=\left\{k_{p}, 0\right\}$, into a signal and idler pair were displayed in Fig.2.3, where the long-time limit, in which both energy and lineal momentum have to be conserved, is depicted as the continuous line. This information is crucial for understanding the many-polariton case that we

discuss from now on. The initial state is taken as $\mathrm{N}$ polaritons pumped at $\mathbf{k}_{\mathbf{p}}=\{0.66,0\} \mu \mathrm{m}^{-1}$. The system is let to evolve and population in $\mathbf{k}$ space is monitored at different times. In Fig.4.5 contour plots of the polariton population distribution are shown, for $\mathrm{N}=1000$ particles initially pumped. Clearly, a strong asymmetry in signal and idler emission patterns is seen. In particular, although there is a small population in the idler modes, it is not comparable to the signal one.

Above the parametric threshold a remarkable different behavior is displayed. As the density of polaritons increases a more significant fraction of the population is concentrated in lowest momentum states, and thus an important intensity is registered in the idler state opposed to the signal one, as can be seen in Fig.4.6. Similar results were reported in Ref.[9]. Furthermore, the finite intensity that rises in the idler state is asyncronous with the evolution of the signal population, in close agreement with experimental results in Ref.[2]. The asymmetry in the shape and time of the formation dynamics of the final state distribution in $\mathbf{k}$ space is a direct result of the different multiple scattering processes affecting signal and idler polaritons. As is well known, for higher momentum states (idler modes), polaritons have a big excitonic component, which implies a stronger coupling with the lattice phonons, and as a consequence a fast decay in idler population. Even though in the time range of the reported dynamics the dominant scattering processes correspond to 

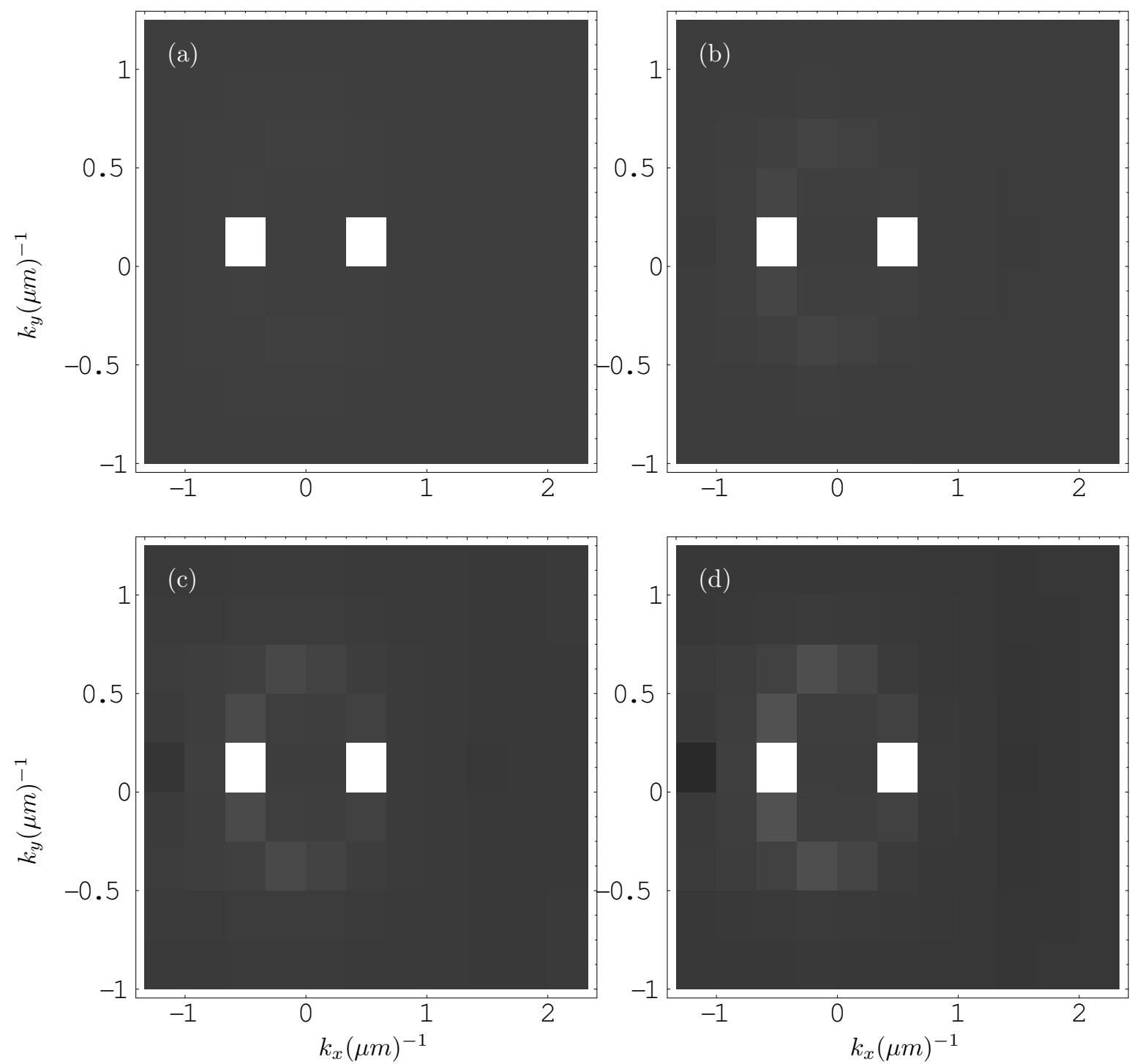

Figure 4.5: Population evolution in momentum space of $\mathrm{N}=1000$ polaritons initially pumped at $\mathbf{k}=\{0.66,0\} \mu \mathrm{m}^{-1}$. The plots correspond to different times: (a) $20 \mathrm{ps}$, (b) $40 \mathrm{ps}$, (c) $60 \mathrm{ps}$ (d) 80 ps, and $10^{4}$ simulations. 
Coulomb interactions, some evidence of decoherence in the idler states, due to the semiconductor environment (phonons), is visible in Fig. 4.6c and 4.6d.

In contrast to mean field approximation, the threshold behavior is well captured by our stochastic method. As the polariton density increases, a dramatic change is observed in the signal population. While below the threshold the signal population is weak, a sudden increase close to the threshold is observed. In Fig.4.7, the signal population is displayed for different times as a function of the number of initially pumped polaritons. Close to $N=2000$, a clear change of slope is registered for any time. The behavior of the idler mode populations (not shown) is analogous.

While part of this dynamics has to be interpreted purely as a bosonic behavior, it is indeed a very interesting result that the coherent rise in population in the signal states is recovered only through the full inclusion of Coulomb correlations, which is done by our method. In this sence, analogous to the bose condensate in atomic physics, the interactions are a source of coherent coupling that leads to macroscopic effects. 

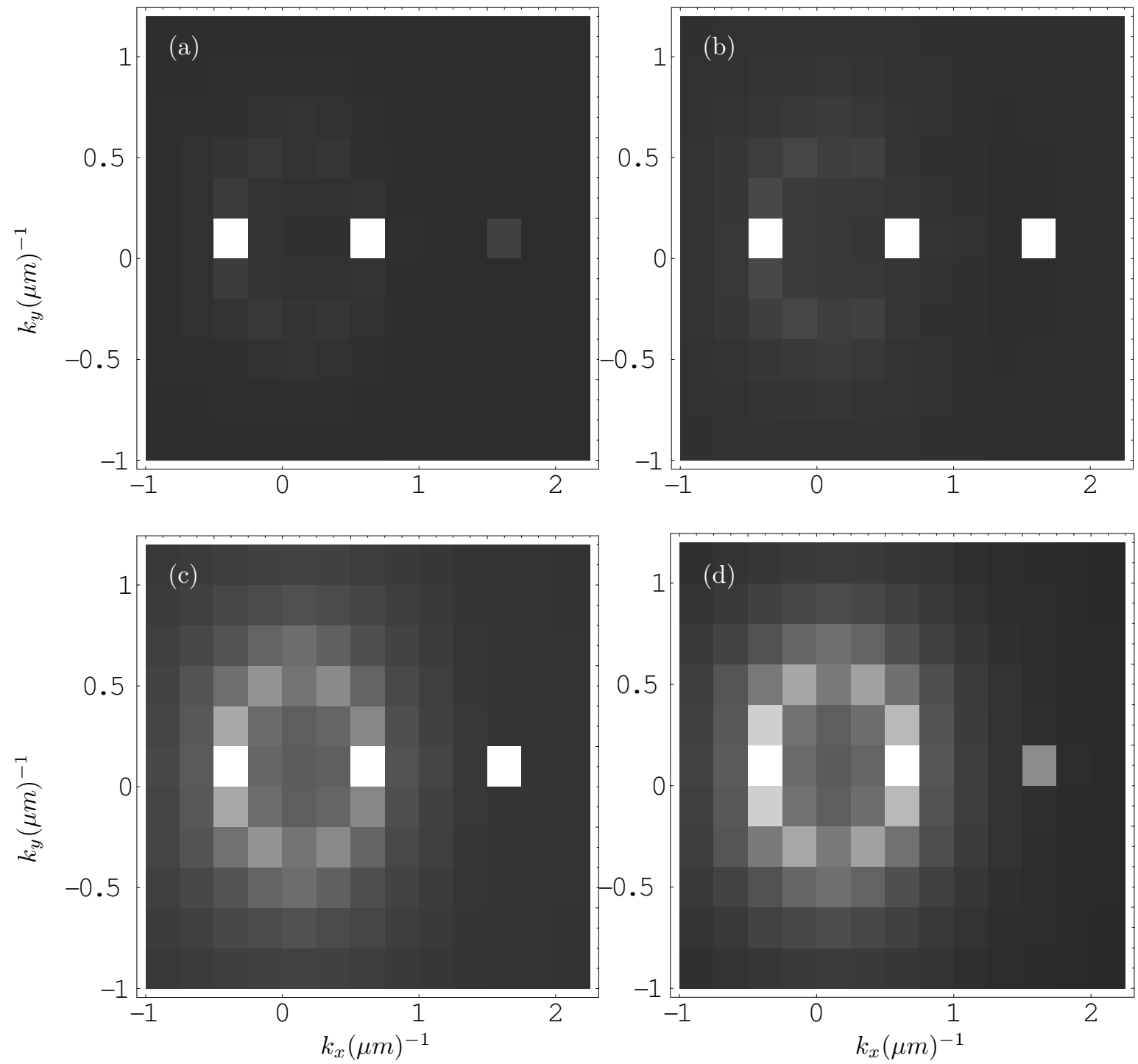

Figure 4.6: Population evolution in the momentum space of $\mathrm{N}=5000$ polaritons initially pumped at $\mathbf{k}=\{0.66,0\} \mu \mathrm{m}^{-1}$. The plots correspond to different times (a) $20 \mathrm{ps}$, (b) $40 \mathrm{ps,} \mathrm{(c)} 60 \mathrm{ps,} \mathrm{(d)}$ $80 \mathrm{ps}$, and $10^{4}$ simulations. 


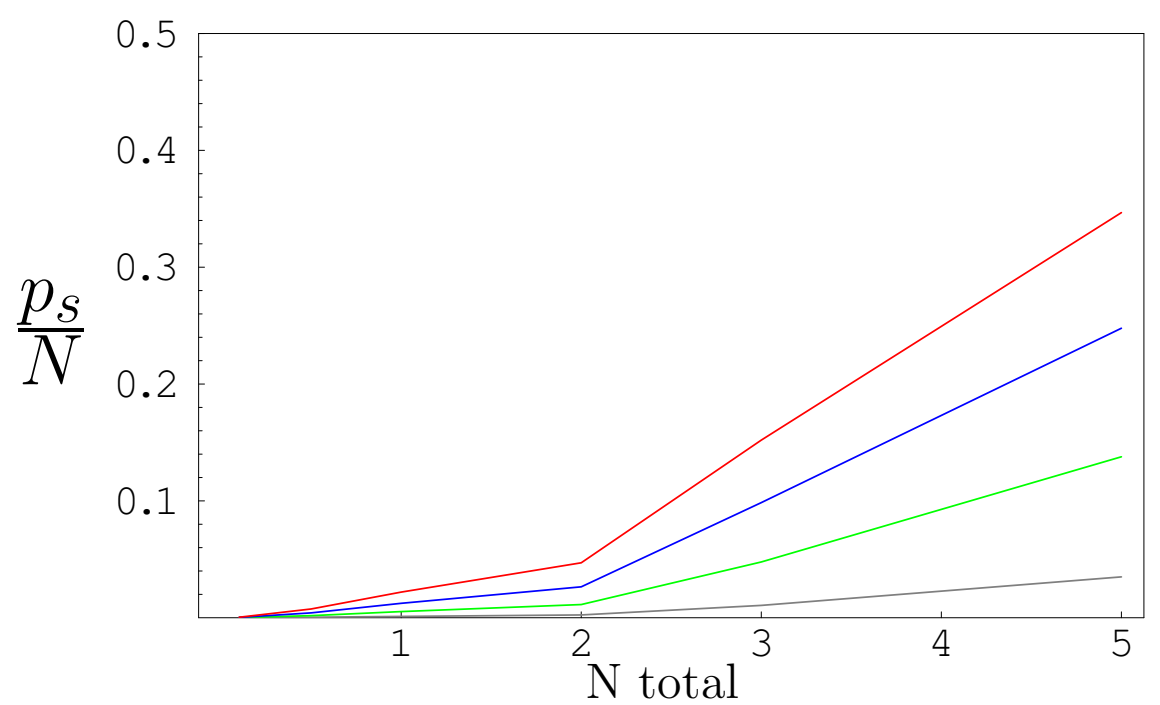

Figure 4.7: Population fraction of the signal mode $\mathbf{k}_{\mathbf{s}}=\{-0.5,0\} \mu m^{-1}$ as a function of the initially pumped polaritons $N$ at different times: $t=5 \mathrm{ps}$ (gray), $t=10 \mathrm{ps}$ (green), $t=15 \mathrm{ps}$ (blue) and $t=20 \mathrm{ps}$ (red). $10^{4}$ realizations for each initial number of total polaritons. 


\section{Chapter 5}

\section{Conclusions}

In the present paper we have presented a stochastic study on the dynamical properties of microcavity polaritons. In particular, we reviewed the dynamical condensation of polaritons emphasizing on the role of Coulomb interactions. Additionally, the threshold behavior of the spontaneous parametric downconversion of polaritons was also discussed. The numerical calculations have been performed by means of stochastic evolution of a reduced state vector in the single particle Hilbert space, separately from the stochastic environment evolution. The resulting stochastic differential equations allows reasonable reduction of the problem which by direct numerical integration of the density matrix would have being cumbersome. For this matter, the stochastic technique turns into a very practical tool for many body problems in condensed matter scenarios. The price to be paid for this formidable reduction of complexity is that a large number of stochastic realizations has to be performed in order to recover the exact solution. The validity of the method was tested and compared positively with previous theoretical and experimental results.

Starting from the cavity photon and the quantum well exciton in the strong coupling regime, we gave a brief guideline in the introduction of the polariton basis. We also discussed the origin of polariton-polariton interactions and the coupling with the semiconductor environment. Along with this theoretical description we underline some of the most important experimental developments in microcavity polaritons which served to us as motivation in the dynamical study of this short living particles.

In the dynamical condensation of polaritons, we found that while the semiconductor environment induces a slow relaxation process, polariton-polariton interactions trigger the sudden development of a macroscopic coherent state characterized by the fact that Coulomb correlations are fully included. This differs clearly from the polariton laser picture, and other recent proposals which claim that this behavior is just a consequence of the particle number conservation [4]. While a more detailed 
study on this phenomenon is needed, our results favor the BEC type phase transition of microcavity polaritons. A definitive proof of the existence of BEC would be a finite value of the order parameter $\left\langle p_{0}\right\rangle$. This would require an extension into a model where the number of polaritons is not conserved.

On the other hand, the dynamical process of parametric emission was studied. There exists an asymmetry in shape and time dependence of the far field emission of signal and idler modes governed by this kind of scattering processes. More specifically, the large excitonic component of the idler polariton is responsible for extra scattering terms, such as the decoherence induced by the coupling with the semiconductor environmnet. The threshold behavior is also well captured by our stochastic method. This corresponds to the exponential rise in signal and idler populations for a given pump density. Coherent properties of this parametric emission process may also be studied by the present method. The latter is an unquestionable interesting field, since the properties of the polaritons is evidenced by the properties of the emmited light after recombination, an important feature for quantum computation. Additionally, a straightforward possibility is to extend the present stochastic method to finite lattice temperature situations.

The main results of this work were presented and in A.E. Pedraza and L. Quiroga [32]. 


\section{Bibliography}

[1] C.Weisbuch et al., Phys.Rev.Lett. 69 (1992) 3314.

[2] W. Langbein, Phys. Rev. B 70 (2004) 205301.

[3] S. Savasta, O. Di Stefano, V. Savona, W. Langbein, Phys. Rev. Lett. 94 (2005) 246401.

[4] F.P. Laussy, G. Malpuech, A. Kavokin, P. Bigenwald, Phys. Rev. Lett. 93 (2004) 016402.

[5] H. Deng et al., Science. 298 (2002) 199.

[6] D. Sarch, V. Savona, cond-mat/0411084 (2004).

[7] I. Carusotto, Y. Castin, J. Dalibard, Phys. Rev. A 63 (2001) 023606.

[8] O.Juillet, Ph. Chomaz, Phys.Rev.Lett. 88 (2002) 142503.

[9] I. Carusotto, C. Ciuti, Phys. Rev. B 72 (2005) 125335.

[10] H.P. Breuer, Phys. Rev. A 69 (2004) 022115.

[11] D. Lacroix, Phys. Rev. A 72 (2005) 013805.

[12] C.Ciuti, P. Schwendimann, A. Quattropani. Phys. Rev. B 63 (2001) 041303

[13] C. Ciuti et al., Semicond. Sci. Technol. 18 (2003) 279.

[14] E. Giacobino et al., Solid State Communications 134 (2005) 97-106.

[15] D. Porras et al., Phys. Rev. B. 66 (2002) 085304.

[16] P.G. Savvidis et al., Phys. Rev. Lett. 84 (2000) 1547.

[17] F.P. Laussy et al., J.Phys.:Condens. Matter 16 (2004) S3665.

[18] F.Tassone, C. Piermarocchi, V. Savona, A. Quattropani, P. Schwendimann, Phys. Rev. B 56 (1997) 7554 .

[19] E.Tokunaga et al,. Phys. Rev B. 63 (2001) 233203. 
[20] G. Malpuech, A. Kavokin, A. Di Carlo, and J.J. Baumberg, Phys. Rev. B 65 (2002) 153310.

[21] L. Banyai, P. Gartner, O.M. Schmitt and H. Haug, Phys. Rev. B 61 (2000) 8823.

[22] L. Banyai and P. Gartner. Phys.Rev. Lett. 88 (2002) 210404.

[23] O.M. Schmitt, D.B. Tran Thoai, P. Gartner, H. Haug, cond-mat/0006040 (2000).

[24] I. Carusotto, I. Castin, Phys. Rev. Lett. 90 (2003) 030401.

[25] O.M. Schmitt et al, Phys.Rev.Lett. 86 (2001) 3839.

[26] M.H. Anderson,J. Ensher, M. Matthews, C. Wieman and E. Cornell, Science 269, (195) 198.

[27] I. Carusotto, Y. Castin, cond-mat/0209135 (2002).

[28] A.E. Pedraza, Bose-Einstein condensation in interacting bosonic systems. Undergraduate Thesis, U. de los Andes (2004).

[29] A.E. Pedraza, L. Quiroga, Rev. Colombiana de Física 35 (2003) 214.

[30] M. Scully and M. Zubairy. Quantum Optics, Cambridge University Press (1997).

[31] J. Wilkie, quant-ph/040739 (2004).

[32] A.E. Pedraza, L. Quiroga, Stochastic dynamics of microcavity polaritons. Solid State Communications, to be published. 